\title{
A Real-Complex Hybrid Modulation Approach for Scaling Up Multiuser MIMO Detection
}

\author{
Juan Carlos De Luna Ducoing, Yi Ma, Senior Member, IEEE, Na Yi, Member, IEEE, and Rahim Tafazolli, Senior \\ Member, IEEE
}

\begin{abstract}
In this paper, a novel approach, namely realcomplex hybrid modulation ( $\mathrm{RCHM})$, is proposed to scale up multiuser multiple-input multiple-output (MU-MIMO) detection with particular concern on the use of equal or approximately equal service antennas and user terminals. By RCHM, we mean that user terminals transmit their data sequences with a mix of real and complex modulation symbols interleaved in the spatial and temporal domain. It is shown, through the system outage probability, RCHM can combine the merits of real and complex modulations to achieve the best spatial diversity-multiplexing trade-off that minimizes the required transmit-power given a sum-rate. The signal pattern of RCHM is optimized with respect to the real-to-complex symbol ratio as well as power allocation. It is also shown that RCHM equips the successive interference canceling MU-MIMO receiver with near-optimal performances and fast convergence in Rayleigh fading channels. This result is validated through our mathematical analysis of the average biterror-rate as well as extensive computer simulations considering the case with single or multiple base-stations.
\end{abstract}

Index Terms-Multiuser multiple-input multiple-output (MUMIMO), detection, real-complex hybrid modulation (RCHM), successive interference cancellation (SIC), widely-linear receiver.

\section{INTRODUCTION}

D ETECTION of multiuser multiple-input multiple-output (MU-MIMO) signals in noise is often modeled as an integer least-squares (ILS) problem, with the goal of minimizing the Euclidean distance between noisy output and the input signal multiplied by a channel transition matrix. Due to the randomness of wireless channels, the MIMO channel matrix can be badly conditioned, and in this case the performance of MU-MIMO detection will be largely degraded. Nevertheless, it has been shown that when the channel matrix has excess number of rows over columns, even a very simple algorithm (i.e. matched filter) becomes near-optimal given the hypothesis of column-wise quasi-orthogonality. This fact has recently motivated the concept of asymmetric MU-MIMO or massive MIMO [1], [2]. Despite their promises, the use of large number of service antennas (and RF chains) over user terminals (UTs) could be an expensive solution. Moreover, linear MU-MIMO detection algorithms have their performances quickly moved away from the optimum with increasing the spatial-domain

This work was supported in part by European Commission under the framework of the FP7 ICT-619555 RESCUE project, and in part by 5G Innovation Centre (5GIC) HEFEC grant. This work has been filed in an international patent with the application No. PCT/GB2017/052012.

The authors are with the Institute for Communication Systems (ICS), University of Surrey, Guildford, England, GU2 7XH, e-mail \{juancarlos.delunaducoing, y.ma, n.yi,r.tafazolli\}@surrey.ac.uk; Tel: +44 1483683609 . user load, and thus they face challenges arising from the application in densely populated areas such as stadiums. All of the above motivate us to revisit the conventional MUMIMO detection problem, with particular concern on equal or approximately equal service antennas and UTs.

In the last two decades, enormous research efforts have been paid towards achieving a good trade-off between the MUMIMO detection performance and computational complexity. Notable contributions include: reduced-complexity sphere decoding [3], [4], lattice-reduction (LR) aided detection and its evolutions (e.g., [5], [6]), successive interference cancellation (SIC or equivalently V-BLAST [7]), semi-definite relaxation [8], likelihood ascent search (LAS) [9], tabu search [10], [11], belief propagation (BP) [12], block-iterative generalized decision feedback equalizer (BI-GDFE) [13] as well as their combinations [6]. A relatively comprehensive review of MUMIMO detection algorithms can be found in the tutorial and survey literature [11], [14].

Despite already remarkable achievements, current MUMIMO technology is still challenged by the signal processing scalability with respect to the size of MU-MIMO networks. It has been shown that most of existing algorithms are too suboptimal for large MIMO systems, and some (e.g. LAS, BP) are near-optimal only for special cases such as MU-MIMO with very large size (e.g. $128 \times 128$ or above) and lower-order modulations (e.g. BPSK). One of the remarkable approaches is the combination of minimum mean-square error (MMSE), LR and SIC algorithms [6], which generally offers near-optimal performances. However, the combined approach features very high computational complexity $\left(5^{\text {th }} \sim 6^{\text {th }}\right.$ order of the MIMO size). Moreover, the MMSE algorithm requires the knowledge of signal-to-noise ratio (SNR), which might be a considerable challenge in interference-limited wireless scenarios.

In light of the literature review, we appreciate that receiver design for MU-MIMO detection is perhaps already a saturated research topic, and thus seek for an alternative solution through joint transmitter and receiver design. Motivated by our earlier work [15], which shows the advantage of using real modulations and a widely linear (WL) receiver for exploiting a much higher spatial diversity-gain at the price of halved spatial multiplexing-gain, the objective of this work is to combine real modulations with spectrum-efficient complex modulations (mainly quadrature amplitude modulation, QAM) to achieve the best spatial diversity-multiplexing trade-off that minimizes the required transmit-power given a sum-rate. Such an approach is named real-complex hybrid modulation (RCHM); and it will be shown that RCHM equips the low- 
complexity MU-MIMO receiver, the combination of WL and SIC, with near-optimal performances. More specifically, major contributions of this paper include:

1) The novel concept of RCHM-MIMO, where UTs transmit their data sequences with a mix of real and complex modulation symbols interleaved in the spatial and temporal domain. The MU-MIMO receiver is suggested to employ the WL-SIC algorithm for channel equalization and detection. For the sake of mitigating error propagation in the SIC procedure, every user's decoded data is verified by cyclic redundancy check (CRC) before interference cancellation; this is the idea reported in [16], [17].

2) The analytical work of RCHM-MIMO system outage probability in Rayleigh-fading channels, which shows the spatial diversity-multiplexing trade-off as a function of realto-complex symbol ratio. Moreover, the outage probability is utilized to optimize the signal pattern of RCHM with the aim of minimizing the required transmit-power given a sum-rate and target outage probability, through configuration of the real-to-complex symbol ratio as well as the power allocation between real and complex symbols.

3) The theoretical analysis of average bit-error-rate (BER) for RCHM-MIMO in Rayleigh-fading channels considering the receiver employs either WL or WL-SIC algorithm. Concerning the exact-BER form for WL-SIC to be mathematically intractable, an approximate-BER form is proposed using a state-machine approach. It is shown that the approximateBER is very close to the BER obtained through computer simulations. Moreover, it is found that the BER of WL-SIC is very close to the maximum likelihood (ML) bound; and it means that the combination of RCHM and WL-SIC yields a near-optimal solution for MU-MIMO detection.

4) The analysis of convergence and computational complexity for the WL-SIC algorithm as far as RCHM-MIMO is concerned. It is shown that RCHM equips the WL-SIC algorithm with fast convergence and low computational complexity, comparable to the linear zero forcing (ZF) algorithm.

Furthermore, extensive computer simulations are presented considering the case with single or multiple base-stations. The performance evaluation involves practical coding and decoding schemes, perfect or estimated channel information, as well as geometric user distribution. The performance of RCHMMIMO is compared with state-of-the art, and the former demonstrates remarkable advantages in terms of both the performance and computational complexity.

The rest of this paper is organized as follows. Section II presents the system model and preliminaries. Section III presents the basic concept of RCHM-MIMO and corresponding transceiver design. Section IV analyzes the average BER and computational complexity of MU-MIMO detection. Computer simulations and discussions are provided in Section $\mathrm{V}$. Section VI draws the conclusion.

\section{System Model AND Preliminaries}

\section{A. Discrete-time Equivalent Model of MU-MIMO Uplink}

Consider MU-MIMO uplink communications, where a set of UTs communicate to the network. It is assumed that the network side has $M$ service antennas simultaneously serving $K$ UTs $(K \leq M)$. It is also assumed that service antennas can fully share their received waveform for joint signal processing, and each UT has a single transmit antenna. This assumption facilitates our technical presentation with the focus on the key novelty and contributions.

The discrete-time equivalent model of MU-MIMO uplink is described into the following matrix form

$$
\mathbf{y}=\mathbf{H x}+\mathbf{v}
$$

where $\mathbf{y}=\left[y_{1}, \ldots, y_{M}\right]^{T}$ stands for the spatial-domain received symbol block, $\mathbf{x}=\left[x_{1}, \ldots, x_{K}\right]^{T}$ for a block of complex symbols with the covariance $\sigma_{x}^{2} \mathbf{I}$, with each symbol selected from a finite constellation set $\mathcal{A}$, with $L=|\mathcal{A}|$ the number of elements in the constellation, $\mathbf{H}$ for the channel transition matrix with the size of $(M) \times(K), \mathbf{v}$ for the additive white Gaussian noise with the covariance $\sigma_{v}^{2} \mathbf{I}$, and the superscript $[\cdot]^{T}$ for the matrix/vector transpose. Taking the large-scale path-loss into account, the channel matrix $\mathbf{H}$ can be decomposed into the Hadamard, or element-wise product of two $(M) \times(K)$ matrices: $\mathbf{H}=\mathbf{G} \circ \mathbf{A}$, where $\mathbf{G}$ represents the small-scale fading of every UT to service-antenna link, and A the large-scale fading (consisting of path loss, shadowing, etc.), whose elements $a_{m, k}$, where $m \in[1, M], k \in[1, K]$, denote the large-scale fading coefficients from the $k^{\text {th }}$ UT to the $m^{\text {th }}$ service antenna. This channel model is flexible in the sense that it can represent link-level $\left(a_{m, k}=1, \forall m, k\right)$, single base station (BS) $\left(a_{m, k}=a_{m^{\prime}, k}, \forall k\right)$, or multiple-BS $\left(a_{m, k}\right.$ depend on the network setup) systems. This work considers the case where all the large-scale fading coefficients in $\mathbf{A}$ are unitary unless otherwise noted. The case of non-normalized A is considered in the multiple base-station scenario simulations in Section V.

\section{B. Preliminaries of $M U-M I M O$ Detection}

The general problem of MU-MIMO detection is easy to state. Given the channel matrix $\mathbf{H}$, how to find the estimate $\hat{\mathbf{x}}$ that minimizes the Euclidean distance through the following objective function

$$
\hat{\mathbf{x}}=\underset{\mathbf{x} \in \mathcal{A}^{K}}{\arg \min }\|\mathbf{y}-\mathbf{H} \mathbf{x}\|
$$

This is known as the classical ILS problem, of which the optimal solution $\hat{\mathbf{x}}$ can be obtained through the ML brute force or sphere decoding algorithm [3]. However, optimal algorithms are very non-linear and computationally inefficient, and they face challenges of practical implementation for future wireless networks with increasing the size of the channel matrix. Moreover, other non-linear algorithms reviewed in Section I are either too expensive in computational cost or too suboptimal in performances.

It is our aim to find an approach that can offer comparable receiver complexity to the linear $\mathrm{ZF}$ algorithm whilst nearoptimal performances. To this end, we revisit the $\mathrm{ZF}$ algorithm, which employs the matrix $\mathbf{W}_{\mathrm{ZF}}=\left(\mathbf{H}^{H} \mathbf{H}\right)^{-1} \mathbf{H}^{H}$ to equalize 
the MIMO channel ${ }^{1}$

$$
\mathbf{z}=\mathbf{W}_{\mathrm{ZF}} \mathbf{y}=\mathbf{x}+\left(\mathbf{H}^{H} \mathbf{H}\right)^{-1} \mathbf{H}^{H} \mathbf{v}
$$

where $[\cdot]^{H}$ stands for the matrix/vector Hermitian transpose. After the channel equalization, every user's message is individually decoded. The complexity of ZF channel equalization is $\mathcal{O}\left(M K^{2}\right)$, and the decoding complexity is $K$ times the single-user decoding complexity. In terms of the performance, the SNR for the $k^{\text {th }}$ element of $\mathbf{z}$ is given by [18]

$$
\gamma_{k}=\gamma_{0} \frac{\operatorname{det}\left(\mathbf{H}^{H} \mathbf{H}\right)}{\operatorname{det}\left(\tilde{\mathbf{H}}^{H} \tilde{\mathbf{H}}\right)}
$$

where $\tilde{\mathbf{H}}$ is formed by removing the $k^{\text {th }}$ column of $\mathbf{H}$ and $\gamma_{0}=\sigma_{x}^{2} / \sigma_{v}^{2}$. Assuming $\mathbf{H}$ to be an i.i.d. complex Gaussian matrix, $\gamma_{k} / \gamma_{0}$ is shown to be chi-square distributed with $2(M-K+1)$ degrees of freedom, i.e. $\gamma_{k} / \gamma_{0} \sim \chi^{2}(2(M-$ $K+1)$ ), and the system outage probability is approximately [19]

$$
\begin{aligned}
\mathcal{P}_{\text {out }}^{(4)} & =1-\mathcal{P}\left(\bigcap_{k=1}^{K}\left\{\mathcal{I}\left(z_{k} ; x_{k} \mid \mathbf{H}\right) \geq \frac{R}{K}\right\}\right) \\
& =1-\left(\mathcal{P}\left(\log \left(1+\gamma_{k}\right) \geq \frac{R}{K}\right)\right)^{K} \\
& \approx K \mathcal{P}\left(\gamma_{k}<2^{R / K}-1\right) \\
& =K F_{\left(\gamma_{k} / \gamma_{0}\right)}\left(\frac{2^{R / K}-1}{\gamma_{0}}\right) \\
& \approx \frac{K\left(2^{R / K}-1\right)^{D_{\text {CPLX }}}}{D_{\text {CPLX }} !} \gamma_{0}^{-D_{\text {CPLX }}},
\end{aligned}
$$

where $R$ is the target system sum-rate and thus $R / K$ is the target data rate of each individual user ${ }^{2}, \mathcal{I}\left(z_{k} ; x_{k} \mid \mathbf{H}\right)$ is the maximum instantaneous mutual information between the $k$-th element of $\mathbf{z}$ and $\mathbf{x}$. Eq.(6) is due to independence among the $K$ streams, (7) is obtained through the first-order Taylor approximation, (8) from the definition of the cumulative distribution function (CDF), (9) from the approximation of the $\mathcal{X}^{2} \mathrm{CDF}$, accurate when the argument is small [20, Eq. (5.66)], and $D_{\mathrm{CPLX}}=M-K+1$ is the spatial diversity order. Comparing to the optimal algorithms with diversity order $M$ (or equivalently, $2 M$ degrees of freedom), the ZF algorithm is too suboptimal in terms of degrees of freedom and spatial diversity order, particularly for the case of $M=K$ (or $M \approx K$ ).

In our recent work [15], UTs are suggested to transmit real symbols in order to achieve a higher spatial diversity-order without sacrificing the computational complexity. Basically, the linear model (1) can be written into a real-signal equivalent

\footnotetext{
${ }^{1}$ The ZF channel equalizer can be trivially replaced by the MMSE channel equalizer. It will be shown that the ZF/WL-SIC receiver is already nearoptimal for RCHM-MIMO, and thus the use of MMSE-SIC receiver offers almost the same performance; but the latter requires an additional computational expense for the SNR estimation. Therefore, in this paper our discussion is focused on the ZF approach due to its simplicity.

${ }^{2}$ Here we consider an equal rate for each UT. As justified in [19], a generalization to unequal rate assignment is immediate, since equal or unequal rate assignments have the same diversity.
}

form

$$
\underbrace{\left[\begin{array}{c}
\Re(\mathbf{y}) \\
\Im(\mathbf{y})
\end{array}\right]}_{=\breve{\mathbf{y}}}=\left[\begin{array}{cc}
\Re(\mathbf{H}) & -\Im(\mathbf{H}) \\
\Im(\mathbf{H}) & \Re(\mathbf{H})
\end{array}\right]\left[\begin{array}{c}
\Re(\mathbf{x}) \\
\Im(\mathbf{x})
\end{array}\right]+\underbrace{\left[\begin{array}{c}
\Re(\mathbf{v}) \\
\Im(\mathbf{v})
\end{array}\right]}_{=\breve{\mathbf{v}}}
$$

Since $\mathbf{x}$ is a real vector, we have $\Im(\mathbf{x})=\mathbf{0}$ and can rewrite (10) into

$$
\breve{\mathbf{y}}=\underbrace{\left[\begin{array}{c}
\Re(\mathbf{H}) \\
\Im(\mathbf{H})
\end{array}\right]}_{=\breve{\mathbf{H}}} \Re(\mathbf{x})+\breve{\mathbf{v}}
$$

Then, the ZF algorithm can be applied onto $\breve{\mathbf{y}}$ with $\mathbf{W}_{\mathrm{ZF}}$ being redefined as: $\mathbf{W}_{\mathrm{ZF}}=\left(\breve{\mathbf{H}}^{H} \mathbf{H}\right)^{-1} \breve{\mathbf{H}}^{H}$, and the channel equalized signal now becomes

$$
\breve{\mathbf{z}}=\Re(\mathbf{x})+\left(\breve{\mathbf{H}}^{H} \breve{\mathbf{H}}\right)^{-1} \breve{\mathbf{H}}^{H} \breve{\mathbf{v}}
$$

The SNR for the $k^{\text {th }}$ element of $\breve{\mathbf{z}}$ takes the same mathematical form as (4), with $\mathbf{H}$ being replaced by $\breve{\mathbf{H}}$, and $\tilde{\mathbf{H}}$ formed by removing the $k^{\text {th }}$ row of $\breve{\mathbf{H}}$. Since $\mathbf{H}$ is an i.i.d. Gaussian matrix with the size $(2 M) \times(K), \gamma_{k}$ can be shown to be chisquare distributed with $(2 M-K+1)$ degrees of freedom [15]. Similar to the derivation of (9), the system outage probability is approximately

$$
\mathcal{P}_{\mathrm{out}}^{(12)} \approx \frac{K\left(2^{2 R / K}-1\right)^{D_{\mathrm{REAL}}}}{\Gamma\left(D_{\mathrm{REAL}}+1\right)} \gamma_{o}^{-D_{\mathrm{REAL}}}
$$

where $D_{\text {REAL }}=M-K / 2+1 / 2$, and $\Gamma(\cdot)$ is the gamma function. It can be concluded that the use of real modulations effectively increases the spatial diversity-order by $(K-1) / 2$ without penalty to the computational complexity. On the other hand, the spatial multiplexing-gain is reduced by $K / 2$ in comparison to the complex modulation.

It is worthwhile to note: 1) The procedure (10)-(12) is well known as the WL processing, which achieves superior performance over strictly linear filters when the signal, interference or noise is improper [21], [22]. In communication systems, the WL algorithm is often employed to process real signals to exploit the signal improperness [15], [23]-[25]. Practical use cases include GSM systems, where the WL algorithm is employed for single-antenna interference cancellation [26][28]. Moreover, WL processing has been utilized in multipleantenna systems including V-BLAST spatial multiplexing [25], [29], space-time codes (STC) [27], [30], [31], and it was shown that the use of improper signaling is able to achieve an increased achievable rate over conventional proper Gaussian signaling for the MIMO interference channel (MIMO-IC) in [32] and for the MISO interference channel (MISO-IC) in [33].

2) The use of real modulations results in performance penalty for the ML optimal algorithms. It is shown that the spatial diversity-order of ML is $M$ regardless of modulations [18], [20], then the approximate system outage probabilities for complex and real modulations using the ML receiver are

$$
\begin{aligned}
& \mathcal{P}_{\text {out }}^{\text {(cplx })} \approx \frac{K\left(2^{R / K}-1\right)^{M}}{M !} \gamma_{0}^{-M}, \\
& \mathcal{P}_{\text {out }}^{\text {(real) }} \approx \frac{K\left(2^{2 R / K}-1\right)^{M}}{M !} \gamma_{0}^{-M} .
\end{aligned}
$$


Denote $\gamma_{(\text {cplx })}$ and $\gamma_{(\text {real })}$ to be $\gamma_{0}$ in the cases of complex and real modulations, respectively. Let $\mathcal{P}_{\text {out }}^{(\mathrm{cplx})}=\mathcal{P}_{\text {out }}^{(\text {real })}$, we can use (14) and (15) to immediately obtain

$$
\frac{\gamma_{(\text {real })}}{\gamma_{(\mathrm{cplx})}}=\frac{2^{2 R / K}-1}{2^{R / K}-1}
$$

which shows the performance penalty in SNR when real modulations are employed in MU-MIMO communications.

As a conclusion of this section, conventional MU-MIMO with complex modulations is not a scalable technology; as already recognized by the technical community (e.g. [20] and many others). The use of real modulations can improve the spatial diversity-gain for linear MU-MIMO receivers at the expense of multiplexing gain. In Section III, we will introduce the concept of RCHM-MIMO, which combines the merits of real and complex modulations to achieve a suitable spatial diversity-multiplexing tradeoff that can largely improve the scalability of MU-MIMO detection.

\section{RCHM-MIMO SYSTEM, DIVERSITY-MULTIPLEXING TRADE-OFF, AND WAVEFORM OPTIMIZATION}

RCHM-MIMO differs from conventional MIMO systems mainly in the sense of waveform design. In the RCHM-MIMO system, every UT transmits a data sequence mixed with real and complex modulation symbols. Such a scheme belongs to the family of hybrid modulations, which include spatial modulation [34], FSK-QAM (F-QAM) [35], interleaving modulation [36], and some others, with each having their special use cases. For instance, the spatial modulation is proposed to increase the multiplexing gain for multi-antenna transmitters with low-cost RF front-end; F-QAM is proposed for celledge interference mitigation; and the interleaving modulation is proposed for blind channel estimation. Here, we stress that RCHM is the hybrid modulation particularly designed for enhancing the scalability of MU-MIMO uplink communications $^{3}$. In this section, we will discuss about the design criteria of RCHM-MIMO, system outage probability, spatial diversitymultiplexing trade-off, as well as the optimization of RCHM waveform.

\section{A. RCHM Waveform and Design Criteria}

Definition 1: Denote $\overline{\mathbf{x}}_{k}=\left[x_{k, 0}, \ldots, x_{k, N-1}\right]^{T}$ to be the temporal symbol block sent by the $k^{\text {th }}$ UT, where $N$ is the length of the block. The spatial symbol block $\mathbf{x}$ in (1) is now labelled by the time index $n \in[0, N-1]$, i.e., $\mathbf{x}_{n}=\left[x_{1, n}, \ldots, x_{K, n}\right]^{T}$. Denote $N_{\mathrm{C}, k}$ and $K_{\mathrm{C}, n}$ to be the number of complex symbols in $\overline{\mathbf{x}}_{k}$ and $\mathbf{x}_{n}$, respectively. RCHM requires

$$
0<N_{\mathrm{C}, k}<N, 0<K_{\mathrm{C}, n}<K, \forall k, n .
$$

Note that RCHM turns into real modulation for $N_{\mathrm{C}, k}=0, \forall k$, or complex modulation for $N_{\mathrm{C}, k}=N, \forall k$.

\footnotetext{
${ }^{3}$ Although this paper considers only uplink MU-MIMO communications, RCHM can be adapted for the MU-MIMO downlink, by taking advantage of the similarity of the objective functions of MIMO detection in the uplink (2) and MIMO precoding with vector perturbation in the downlink [37], and replacing the WL equalizer with a WL precoder.
}

As far as user fairness and signalling overhead are concerned, the RCHM waveform design should follow three criteria:

C1) Every spatial block $\mathbf{x}_{n}, \forall n$, shall employ the same realto-complex symbol ratio, i.e., $\alpha_{n}=\left(K-K_{\mathrm{C}, n}\right) /\left(K_{\mathrm{C}, n}\right)$ or equivalently, $K_{\mathrm{C}, n}$ shall be identical for $n \in[1, N]$. Such can largely simplify the RCHM-MIMO system design and optimization. The notations $\alpha_{n}$ and $K_{\mathrm{C}, n}$ can be simplified by omitting the subscript $[\cdot]_{n}$.

C2) For the sake of user fairness, UTs should be equal for sharing the spatial degrees of freedom. It means that the real-to-complex symbol ratio should be identical for all users, i.e., $\beta_{k}=\left(N-N_{\mathrm{C}, k}\right) /\left(N_{\mathrm{C}, k}\right)$ or equivalently, $N_{\mathrm{C}, k}$ shall be identical for $k \in[1, K]$.

C3) UTs can have different pattern to place their real and complex symbols in the time-domain block $\overline{\mathbf{x}}_{k}$ in order to fulfill the criterion $C 2$ ). Then, the network will have to inform UTs regarding the pattern for them to use. The set of patterns should be made as small as possible so as to minimize the signalling overhead. Please see Section $\mathrm{V}$ for detailed discussion.

Lemma 1: Given $M$ the maximum of active UTs, a sufficient condition for the criteria $C 1)-C 2$ ) to be satisfied is: $N$ is a common multiple of the integer set $K \in\{1,2, \ldots, M\}$.

Proof: Denote $K_{\mathrm{C}}^{\star}$ to be the optimum of $K_{\mathrm{C}}$ given $K$. The criterion $C 1)$ requires every spatial block to utilize the optimum real-to-complex ratio $\alpha^{\star}=\left(K-K_{\mathrm{C}}^{\star}\right) / K_{\mathrm{C}}^{\star}$. According to $C 2$ ), every UT should have

$$
N_{\mathrm{C}}=\left(N K_{\mathrm{C}}^{\star}\right) / K
$$

where we omit the subscript $[\cdot]_{k}$ for notation simplicity. Since $N_{\mathrm{C}}$ is an integer, it is necessary that $\left(N K_{\mathrm{C}}^{\star}\right)$ is a multiple of $K$ to satisfy (18). This necessary condition is a bit strict since $K_{\mathrm{C}}^{\star}$ is a function of $K$; as shown in Section III-C. We note that $K_{\mathrm{C}}^{\star}$ is also an integer, hence (18) is still satisfied if $N$ is a common multiple for $K \in\{1,2, \ldots, M\}$.

When the length of temporal symbol block is configured according to Lemma 1, it is trivial to find the result $\alpha^{\star}=\beta^{\star}$. Then, the optimization procedure is simplified by determining $\alpha^{\star}$ or $K_{\mathrm{C}}^{\star}$ only. In Sections III-B and III-C, we will introduce the design and optimization of RCHM-MIMO with the criteria C1)-C3) to be satisfied.

\section{B. Outage Probability and Spatial Diversity-Multiplexing Trade-off with ZF Equalizer}

Consider the MU-MIMO receiver employing the ZF algorithm for the MIMO channel equalization. The criterion C1) requires every spatial block $\mathbf{x}_{n}$ to use the same real-tocomplex symbol ratio $\alpha$, hence our outage probability analysis can ignore the subscript $[\cdot]_{n}$.

Given $K_{\mathrm{C}}$ complex symbols within $\mathbf{x}$, the term $\Re(\mathbf{x})$ in (10) remains a $K \times 1$ real vector, and $\Im(\mathbf{x})$ has $K_{\mathrm{C}}$ nonzero real elements. In order to simplify the notation, we denote $\overline{\Im(\mathbf{x})}$ to be a $K_{\mathrm{C}} \times 1$ vector containing only nonzero elements. In addition, we let $\breve{\mathbf{H}}_{1}=\breve{\mathbf{H}}$ and $\breve{\mathbf{H}}_{2}$ a $(2 M) \times\left(K_{\mathrm{C}}\right)$ matrix formed by collecting $K_{\mathrm{C}}$ columns of 
the matrix $\left[-\Im(\mathbf{H})^{T}, \Re(\mathbf{H})^{T}\right]^{T}$ corresponding to $\overline{\Im(\mathbf{x})}$. The real-signal equivalent model (10) reads as

$$
\breve{\mathbf{y}}=\left[\breve{\mathbf{H}}_{1} \breve{\mathbf{H}}_{2}\right]\left[\frac{\Re(\mathbf{x})}{\Im(\mathbf{x})}\right]+\breve{\mathbf{v}} .
$$

This linear model shares the same form as the conventional complex-modulated signal model, and thus all of existing advanced MIMO detection algorithms such as ML, sphere decoding, LR, LAS, ZF-SIC (or equivalently WL-SIC for real signals) can be straightforwardly applied to (19). We appreciate the nature of low computational complexity for the WL-SIC algorithm, and will show its near-optimality when handling the signal model (19).

Let us define $\overline{\mathbf{H}}=\left[\begin{array}{ll}\breve{\mathbf{H}}_{1} & \breve{\mathbf{H}}_{2}\end{array}\right]$. The output of ZF channel equalizer is

$$
\breve{\mathbf{z}}=\left[\frac{\Re(\mathbf{x})}{\Im(\mathbf{x})}\right]+\left(\overline{\mathbf{H}}^{H} \overline{\mathbf{H}}\right)^{-1} \overline{\mathbf{H}}^{H} \breve{\mathbf{v}}
$$

with dimensions $\left(K+K_{\mathrm{C}}\right) \times 1$. Furthermore, we denote $\tilde{\mathbf{z}}$ to be the resulting $2 K \times 1$ vector after reinserting the zeros to the vector $\breve{\mathbf{z}}$ in the positions that were removed when $\overline{\Im(x)}$ was formed. Moreover, the equalized signal can then be reconstructed into its complex form, denoted as $\overline{\mathbf{z}}$, with dimensions $K \times 1$, with $\overline{\mathbf{z}}=\tilde{\mathbf{z}}_{[1, K]}+j \tilde{\mathbf{z}}_{[K+1,2 K]}$, where $\tilde{\mathbf{z}}_{[1, K]}$ and $\tilde{\mathbf{z}}_{[K+1,2 K]}$ denote the first and second half, respectively, of the elements of $\tilde{\mathbf{z}}$.

Denote $\bar{z}_{k}$ to be the $k^{\text {th }}$ element of $\overline{\mathbf{z}}$, and $k_{\mathrm{R}}$ and $k_{\mathrm{C}}$ the indices of those elements that correspond to real and complex signals, respectively. The SNR for $\bar{z}_{k_{\mathrm{R}}}$ (the elements of corresponding to the real-valued streams with zero imaginary part) is $\mathcal{X}^{2}$-distributed with $\left(2 M-K-K_{\mathrm{C}}+1\right)$ degrees of freedom. However, the SNR for the real or imaginary components of the complex streams $\bar{z}_{k_{\mathrm{c}}}$ does not exactly follow $\mathcal{X}^{2}$-distribution but approximately so. Since the real and imaginary component can exchange their positions within a complex symbol, the components of $\bar{z}_{k_{\mathrm{c}}}$ have $\left(2 M-K-K_{\mathrm{C}}+2\right)$ degrees of freedom. Given that the elements of $\overline{\mathbf{z}}$ follow two different distributions, the performance analysis used for real-modulated MU-MIMO or complex-modulated MU-MIMO having a single distribution cannot be straightforwardly applied to the RCHM-MIMO system.

Lemma 2: Given equal rate for each individual UT, the system outage probability of RCHM-MIMO with the ZF channel equalizer is approximately

$$
\begin{aligned}
\mathcal{P}_{\text {out }}^{(20)} \approx( & \frac{\left(K-K_{\mathrm{C}}\right)\left(2^{2 R /\left(K+K_{\mathrm{c}}\right)}-1\right)^{D_{\text {Rснм }}}}{\Gamma\left(D_{\text {RCHм }}+1\right)} \\
& \left.+\frac{K_{\mathrm{C}}\left(2^{2 R /\left(K+K_{\mathrm{C}}\right)}-1\right)^{\left(D_{\text {Rснм }}+\frac{1}{2}\right)}}{\Gamma\left(D_{\mathrm{RCHM}}+\frac{3}{2}\right) \sqrt{\gamma_{o}}}\right) \gamma_{o}^{-D_{\text {Rснм }}}
\end{aligned}
$$

where $D_{\text {RCнм }}=\left(2 M-\left(K+K_{\mathrm{C}}-1\right)\right) / 2$ is the spatial diversityorder of RCHM-MIMO.

Proof: For real-modulated signals, the maximum mutual information between $\bar{z}_{k_{\mathrm{R}}}$ and $x_{k_{\mathrm{R}}}$ is: $\mathcal{I}_{\mathrm{R}}(k)=\frac{1}{2} \log \left(1+\gamma_{k_{\mathrm{R}}}\right)$, where $\gamma_{k_{\mathrm{R}}} / \gamma_{0}$ is chi-square distributed with $\left(2 M-K-K_{\mathrm{C}}+1\right)$ degrees of freedom; the complex-modulated signals carry twice the rate of real signals, therefore the maximum mutual information for the $k_{\mathrm{C}}$-th complex modulated signals is:

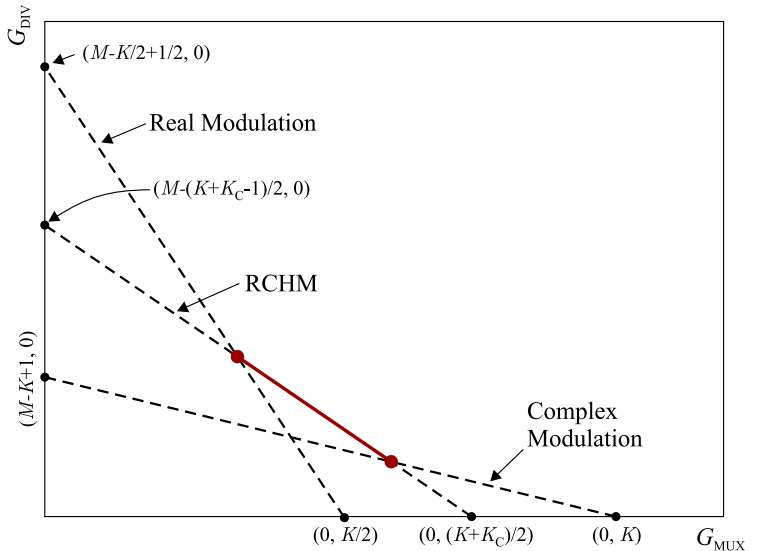

Fig. 1. The spatial diversity-multiplexing trade-off $\left(G_{\mathrm{DIV}}, G_{\mathrm{MUX}}\right)$ for real complex and RCHM modulated MIMO using the ZF channel equalizer.

$\mathcal{I}_{\mathrm{C}}(k)=\log \left(1+\gamma_{k_{\mathrm{c}}}\right)$, where $\gamma_{k_{\mathrm{c}}} / \gamma_{0}$ is approximately $\mathcal{X}^{2}$ distributed with $\left(2 M-K-K_{\mathrm{C}}+2\right)$ degrees of freedom. Given the system target rate $R$ and equal rate assignment for each element of $\breve{\mathbf{z}}: R_{o}=R /\left(K+K_{\mathrm{C}}\right)$, the system outage probability is the probability for at least one of UTs to be outage

$$
\begin{aligned}
\mathcal{P}_{\text {out }}^{(20)} & =1-\left(\mathcal{P}\left(\mathcal{I}_{\mathrm{R}} \geq R_{o}\right)\right)^{K-K_{\mathrm{C}}}\left(\mathcal{P}\left(\mathcal{I}_{\mathrm{C}} \geq 2 R_{o}\right)\right)^{K_{\mathrm{C}}} \\
& \approx\left(K-K_{\mathrm{C}}\right) \mathcal{P}\left(\mathcal{I}_{\mathrm{R}}<R_{o}\right)+K_{\mathrm{C}} \mathcal{P}\left(\mathcal{I}_{\mathrm{C}}<2 R_{o}\right)
\end{aligned}
$$

In (22), we omit the user index for the mutual information $\mathcal{I}_{R}$ and $\mathcal{I}_{C}$ since they are user independent in the outage probability computation. Moreover, the approximation in (23) is accurate when the outage probabilities for each element are small. Following the procedure in [20], it is trivial to derive from (23) to (21).

Lemma 2 shows that the spatial diversity-order of RCHMMIMO is $D_{\text {RCHM }}$, which fulfills: $D_{\text {REAL }}>D_{\text {RCHM }}>D_{\text {CPLX }}$. Moreover, it is easily understood that the spatial multiplexingorder of RCHM-MIMO is: $\left(K+K_{\mathrm{C}}\right) / 2$, which is also in between of real modulations $(K / 2)$ and complex modulations (K). According to [20], [38], the spatial diversity-multiplexing trade-off of RCHM-MIMO is given by

$$
G_{\text {DIV }}=D_{\text {Rснм }}\left(1-\frac{G_{\text {MUX }}}{\left(K+K_{\mathrm{C}}\right) / 2}\right)
$$

where $G_{\mathrm{DIV}}$ and $G_{\mathrm{MUX}}$ stand for the diversity gain and multiplexing gain, respectively. Eqn. (24) is plotted in Fig. 1 together with the spatial diversity-multiplexing trade-off of real and complex modulations. The solid line shows the range where RCHM-MIMO outperforms the real-MIMO and complex-MIMO in terms of both the spatial diversity-gain and multiplexing-gain.

\section{RCHM Waveform Design and Optimization}

The objective of RCHM waveform design and optimization involves power allocation between real and complex symbols and real-to-complex ratio optimization, which will be mainly based on the system outage probability. Moreover, the waveform should be designed to minimize the signalling overhead as well as computational complexity. 
1) Power Allocation: The system outage probability (21) implicitly assumes that the SNR is identical for both real and complex symbols, or equivalently a complex symbol doubles the transmit-power of a real symbol. When the SNR is different for real and complex symbols, the system outage probability reads as (cf. (21))

$$
\begin{aligned}
\mathcal{P}_{\text {out }}^{(20)} \approx & \frac{\left(K-K_{\mathrm{C}}\right)\left(2^{2 R /\left(K+K_{\mathrm{C}}\right)}-1\right)^{D_{\text {RСн }}}}{\Gamma\left(D_{\mathrm{RCHM}}+1\right)} \gamma_{\mathrm{R}}^{-D_{\text {Rснм }}} \\
& +\frac{K_{\mathrm{C}}\left(2^{2 R /\left(K+K_{\mathrm{C}}\right)}-1\right)^{\left(D_{\mathrm{RCHм}}+\frac{1}{2}\right)}}{\Gamma\left(D_{\mathrm{RCHM}}+\frac{3}{2}\right)} \gamma_{\mathrm{C}}^{-\left(D_{\mathrm{RCHM}}+\frac{1}{2}\right)}
\end{aligned}
$$

where $\gamma_{R}$ and $\gamma_{C}$ are the transmit-power to noise ratio for real and complex symbols, respectively, with the relationship

$$
\left(K-K_{\mathrm{C}}\right) \gamma_{\mathrm{R}}+\left(2 K_{\mathrm{C}}\right) \gamma_{\mathrm{C}}=\left(K+K_{\mathrm{C}}\right) \gamma_{o}
$$

where $\left(K+K_{\mathrm{C}}\right) \gamma_{o} \sigma_{v}^{2}$ is the total transmit-power constraint The objective of power allocation is to solve

$$
\gamma_{\mathrm{R}}^{\star}=\underset{\gamma_{\mathrm{R}}}{\arg \min } \mathcal{P}_{\text {out }}^{(20)}, \text { s.t. (26) }
$$

Theorem 1: Suppose $D_{\mathrm{RCHM}}$ to be sufficiently large (e.g. $D_{\text {RCHм }} \gg 1$ ), the power allocation between real and complex symbols, s.t. (26), should fulfill the condition

$$
\frac{\gamma_{\mathrm{C}}}{\gamma_{\mathrm{R}}} \approx\left(\frac{1}{2 \eta \sqrt{D_{\mathrm{RCHM}}+1}}\right)^{\frac{1}{D_{\mathrm{RCHM}}+1}}
$$

where $\eta=\sqrt{\gamma_{\mathrm{C}}} / \sqrt{2^{\left(2 R /\left(K+K_{\mathrm{c}}\right)\right)}-1}$.

Proof: It is not hard to understand that $\mathcal{P}_{\text {out }}^{(20)}$ in $(25)$ is a convex function of $\gamma_{\mathrm{R}}$. Hence, the minimum of $\mathcal{P}_{\text {out }}^{(20)}$ is achieved when $\left(\partial \mathcal{P}_{\text {out }}^{(20)}\right) /\left(\partial \gamma_{\mathrm{R}}\right)=0$. It is tedious but straightforward to solve the equation and obtain

$\frac{\gamma_{\mathrm{C}}^{\left(D_{\text {Rснм }}+\frac{3}{2}\right)}}{\gamma_{\mathrm{R}}^{\left(D_{\mathrm{RCHM}}+1\right)}}=\frac{\left(D_{\mathrm{RCHM}}+\frac{1}{2}\right) \Gamma\left(D_{\mathrm{RCHM}}+1\right) \sqrt{2^{\left(2 R /\left(K+K_{\mathrm{C}}\right)\right)}-1}}{2 D_{\mathrm{RCHM}} \times \Gamma\left(D_{\mathrm{RCHM}}+\frac{3}{2}\right)}$

Given $D_{\mathrm{RCHM}} \gg 1$, (29) can be simplified by using a good approximation: $\left(D_{\mathrm{RCHM}}+\frac{1}{2}\right) \approx D_{\mathrm{RCHM}}$. Moreover, the property of Gamma function: $\Gamma\left(D_{\mathrm{RCHM}}+\frac{3}{2}\right) \approx \Gamma\left(D_{\mathrm{RCHM}}+1\right) \sqrt{D_{\mathrm{RCHM}}+1}$ is also a good approximation for $D_{\mathrm{RCHM}} \gg 1$. Applying these two approximations in (29) immediately leads to (28).

Considering RCHM-MIMO often operating in a high diversity order, we can use the following limit to further simplifying (28)

$$
\frac{\gamma_{\mathrm{C}}}{\gamma_{\mathrm{R}}}=\lim _{D_{\text {Rснм }} \rightarrow \infty}\left(\frac{1}{2 \eta \sqrt{D_{\text {RCHM }}+1}}\right)^{\frac{1}{D_{\text {RСнм }}+1}}=1
$$

which suggests a complex symbol double the transmit power of a real symbol for RCHM-MIMO systems with a high diversity-order (e.g. $D_{\mathrm{RCHM}}>5$ ), and in this case we again have $\gamma_{\mathrm{R}}=\gamma_{\mathrm{C}}=\gamma_{\mathrm{o}}$.

2) Real-to-Complex Symbol Ratio Optimization: Suppose $D_{\mathrm{RCHM}}$ to be large enough to enable the condition $\gamma_{\mathrm{R}}=\gamma_{\mathrm{C}}=$ $\gamma_{o}$, we can utilize the outage probability (21) to study the real-to-complex symbol ratio. With the approximation used in

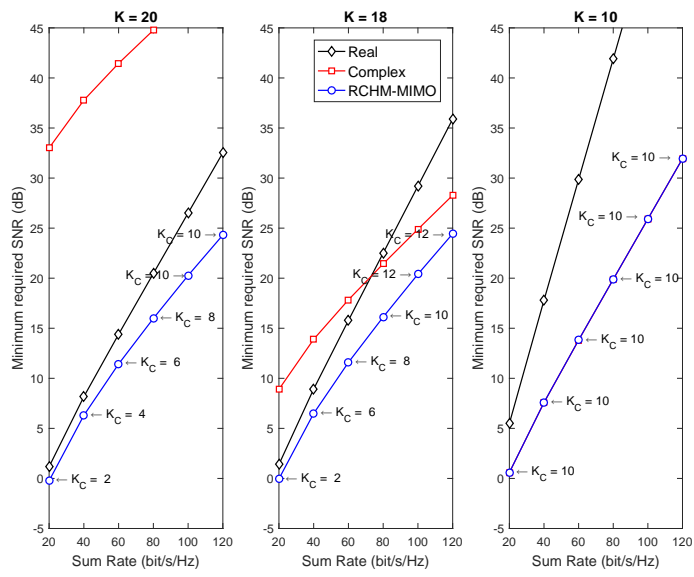

Fig. 2. Minimum SNR required for RCHM-MIMO to achieve a $1 \%$ outage probability, for real, complex and RCHM modulation. The plots are for a system with 20 BS antennas and three system loading scenarios with $K=$ $\{20,18,10\}$.

the proof of Theorem 1, the outage probability (21) can be simplified into

$$
\begin{aligned}
& \mathcal{P}_{\text {out }}^{(20)} \approx\left(\frac{\left(K-K_{\mathrm{C}}\right) \eta \sqrt{D_{\mathrm{RCHM}}+1}+K_{\mathrm{C}}}{\eta \Gamma\left(D_{\mathrm{RCHM}}+1\right) \sqrt{D_{\mathrm{RCHM}}+1}}\right) \\
& \times\left(2^{2 R /\left(K+K_{\mathrm{C}}\right)}-1\right)^{D_{\mathrm{RCHM}}} \gamma_{o}^{-D_{\mathrm{RCHм}}},
\end{aligned}
$$

or reversely

$$
\begin{gathered}
\gamma_{o} \approx\left(2^{2 R /\left(K+K_{\mathrm{C}}\right)}-1\right) \\
\left(\frac{\left(K-K_{\mathrm{C}}\right) \eta \sqrt{D_{\mathrm{RCHM}}+1}+K_{\mathrm{C}}}{\mathcal{P}_{\text {out }}^{(20)} \eta \Gamma\left(D_{\mathrm{RCHM}}+1\right) \sqrt{D_{\mathrm{RCHM}}+1}}\right)^{\frac{1}{D_{\text {Rснм }}}}
\end{gathered}
$$

Theorem 2: Given the outage probability $\mathcal{P}_{\text {out }}^{(20)}, M, K$, the optimal real-to-complex ratio is achieved through

$$
K_{\mathrm{C}}^{\star}=\underset{K_{\mathrm{C}}}{\arg \min } \gamma_{o}
$$

and $\alpha^{\star}=\left(K-K_{\mathrm{C}}^{\star}\right) / K_{\mathrm{C}}^{\star}$.

We recognize that $(32)$ is mathematically intractable, and thus employ a numerical approach to study the optimum ratio (33). First of all, our numerical results (omitted due to limited space) have shown that $\gamma_{o}$ is a convex function of $K_{\mathrm{C}}$ given the parameters $\mathcal{P}_{\text {out }}^{(20)}, M, K$. Based on this observation, we can utilize (32) to find the minimum $\gamma_{o}$ as a function of the system overall rate $R$; as a numerical example shown in Fig. 2 .

This numerical example delivers some interesting implications:

i) In fully-loaded systems $(M=K=20)$, the conventional complex-modulated MU-MIMO always performs the worst. There is at least $20 \mathrm{~dB}$ SNR difference between the conventional MU-MIMO and the RCHM-MIMO. This is not surprising as conventional MU-MIMO is not scalable in the fully-loaded case. The real-modulated MU-MIMO proposed in [15] offers very close SNR to the RCHM-MIMO at low data rate (e.g. $40 \mathrm{bit} / \mathrm{s} / \mathrm{Hz}$ or lower), and the SNR gap grows quickly with the increase of data rate. This is 
TABLE I

DiVERSITY, PAPR, AND \# OF INVERSIONS FOR A $20 \times 20$ MU-MIMO with MODULATION SCHEMES WITH AN AVERAGE OF 6 BIT/SYMBOL (SUM-RATE OF $120 \mathrm{BIT} / \mathrm{S} / \mathrm{Hz})$

\begin{tabular}{cccc}
\hline RCHM Scheme & Diversity & PAPR & \# Channel Inversions \\
\hline Case 1: RCHM 16ASK/256QAM, $K_{\mathrm{C}} / K=0.5$ & 5.5 & $2.73(4.4 \mathrm{~dB})$ & 2 \\
Case 2: null/256QAM, $K_{\mathrm{C}} / K=0.75$ & 6 & $3.53(5.5 \mathrm{~dB})$ & 4 \\
\hline
\end{tabular}

also expected since RCHM-MIMO is designed to achieve the best spatial diversity-multiplexing trade-off by configuring $K_{\mathrm{C}}$. It is observed that $K_{c}^{\star}$ is small at the low data rate $\left(K_{c}^{\star} / K=10 \%\right)$, and it generally grows with the increase of data rate. This clearly indicates that RCHM takes more advantages from complex modulation at higher data rates, and from real modulations at lower data rates.

ii) In heavily-loaded systems $(M=20, K=18)$, the SNR gap between the conventional MU-MIMO and RCHM-MIMO reduces to $3-5 \mathrm{~dB}$, and the gap between the real-modulated MU-MIMO and RCHM-MIMO gets largely increased (up to $13 \mathrm{~dB}$ at the data rate of $120 \mathrm{bit} / \mathrm{s} / \mathrm{Hz})$. Again, $K_{\mathrm{C}}^{\star} / K$ generally increases from $22.2 \%$ to $77.8 \%$ with the increase of data rate.

iii) In lightly-loaded systems ( $M=20, K=10)$, the spatial multiplexing-gain becomes more important than the spatial diversity-gain. This is the case where the real-modulated MUMIMO performs the worst, and RCHM-MIMO switches to the complex-modulated MU-MIMO (i.e. $K_{\mathrm{C}}^{\star} / K=100 \%$ in most of cases).

Remark 1: The optimization so far is based on the outage probability (25), which assumes the doubled rate for complex symbols over real symbols. More generally, we define a ratio $\beta=R_{\mathrm{C}} /\left(2 R_{o}\right)$ with $R_{\mathrm{C}}$ denoting the rate for complex symbols. Then, the outage probability (25) becomes

$$
\begin{aligned}
\mathcal{P}_{\mathrm{out}}^{(20)} \approx & \frac{\left(K-K_{\mathrm{C}}\right)\left(2^{2 R_{\mathrm{R}}}-1\right)^{D_{\text {Rснм }}}}{\Gamma\left(D_{\mathrm{RCHM}}+1\right)} \gamma_{\mathrm{R}}^{-D_{\text {Rснм }}} \\
& +\frac{K_{\mathrm{C}}\left(2^{2 \beta R /\left(K+K_{\mathrm{C}}\right)}-1\right)^{\left(D_{\mathrm{RCHм}}+\frac{1}{2}\right)}}{\Gamma\left(D_{\mathrm{RCHM}}+\frac{3}{2}\right)} \gamma_{\mathrm{C}}^{-\left(D_{\text {Rснм }}+\frac{1}{2}\right)}
\end{aligned}
$$

where $R_{\mathrm{R}}=R\left(K+K_{\mathrm{C}}(1-2 \beta)\right) /\left(K^{2}-K_{\mathrm{C}}^{2}\right)$. This result renders (27) and (33) a complex multi-parameter optimization problem. Nevertheless, we are able to numerically study the optimization issue, and find the best performance achieved at: $R_{\mathrm{R}}=0, \gamma_{\mathrm{R}}=0$; and the optimum ratio $K_{\mathrm{C}} / K$ varies with respect to $K$ and $R$; similar to the result depicted in Fig. 2. It suggests the use of null real symbols for the RCHMMIMO system. However, we argue that the use of null real symbols largely increases the peak-to-average power ratio (PAPR), which is a considerable problem particularly for the uplink power efficiency. Table I compares the cases with or without using the null real symbol given the sum rate of 120 $\mathrm{bit} / \mathrm{s} / \mathrm{Hz}, \beta$ and the power allocation; both are optimized in terms of the ratio $K_{\mathrm{C}} / K$. Given the comparable diversityorder (performance), Case 2 shows much higher PAPR than Case 1. In fact, the PAPR difference increases with the sum rate since Case 2 has considerably fewer temporal degrees-offreedom for the power loading. The other remarkable issue is that Case 2 costs doubled complexity for the channel inverse in comparison with Case 1. This is because the ratio $K_{\mathrm{C}} / K=0.5$ only introduces two different RCHM patterns, and $K_{\mathrm{C}} / K=0.75$ introduces four different RCHM patterns (see the discussion on RCHM pattern in Section V-A).

\section{BER ANALYSIS FOR RCHM-MIMO WITH ASK-QAM MODULATION AND ZF-SIC RECEIVER}

The theoretical work in Section III is implicitly based on the assumption of real and complex modulations with Gaussian codebook, and the detection algorithm after the ZF channel equalization is assumed to be optimum. In this section, the real and complex modulations are replaced by $L_{\mathrm{R}}$-ary amplitude shift keying $\left(L_{\mathrm{R}}-\mathrm{ASK}\right)$ and $L_{\mathrm{C}}$-ary $L_{\mathrm{C}}$-QAM, respectively, and the receiver employs the ZF-SIC algorithm. We will investigate the average BER for ASK-QAM modulated MU-MIMO in i.i.d. Rayleigh-fading channels, and the theoretical result will be confirmed by simulation results presented in Section V.

\section{A. Briefing The Transmitter-Receiver Chain}

The signal model of ASK-QAM MU-MIMO does not have fundamental difference to the RCHM signal model in Section III. Apart from using practical modulation schemes, every UT employs CRC to enable error detection at the receiver side. Practical forward error correction (FEC) codes can be utilized to protect the message.

At the receiver side, the ZF-SIC detection procedure is summarized as follows:

s1) Perform the ZF channel equalization on $\breve{\mathbf{y}}$ in (19);

s2) Perform detection on the output of the channel equalizer $\breve{\mathbf{z}}$ in (20). This step can be conducted in parallel for a receiver supporting parallel computing;

s3) Perform CRC check on every detected data sequences. Those passed CRC will be subtracted from the received signal $\breve{\mathbf{y}}$;

s4) Repeat s1)-s3) until: 1) all sequences are successfully reconstructed, or 2) no new sequence is successfully reconstructed in the current iteration.

This algorithm is not novel, and it was originally reported for MIMO detection with QAM modulations [16], [17].

\section{B. Average BER Analysis for ZF-SIC Receiver with ASK-QAM Hybrid Modulation}

Our BER analysis starts from the initial state of the ZF-SIC process, i.e., the first attempt of signal detection right after the ZF channel equalization. Considering an ASK-QAM sequence consisting of $N_{\mathrm{R}}$ ASK symbols and $N_{\mathrm{C}}$ QAM symbols, the 
average BER of ASK-QAM for the initial state, denoted by $\overline{\mathcal{P}}_{e, \mathrm{AQAM}}^{(0)}$, is simply the weighted average

$$
\overline{\mathcal{P}}_{e, \mathrm{AQAM}}^{(0)}=\frac{N_{\mathrm{R}} \log _{2}\left(L_{\mathrm{R}}\right) \overline{\mathcal{P}}_{e, \mathrm{ASK}}^{(0)}+N_{\mathrm{C}} \log _{2}\left(L_{\mathrm{C}}\right) \overline{\mathcal{P}}_{e, \mathrm{QAM}}^{(0)}}{N_{\mathrm{R}} \log _{2}\left(L_{\mathrm{R}}\right)+N_{\mathrm{C}} \log _{2}\left(L_{\mathrm{C}}\right)}
$$

where $\overline{\mathcal{P}}_{e, \mathrm{ASK}}^{(0)}$ and $\overline{\mathcal{P}}_{e, \mathrm{QAM}}^{(0)}$ denote the average BER for ASK and QAM, respectively.

Given the instantaneous SNR $\rho_{k}$ for the $k^{\text {th }} L_{\mathrm{R}}$-ASK symbol within $\breve{\mathbf{z}}$ in (20), the instantaneous BER of $L_{\mathrm{R}}$-ASK is given by [39]

$$
\mathcal{P}_{e, \mathrm{ASK}}^{(0)}\left(\rho_{k}\right) \approx \frac{1}{\log _{2} L_{\mathrm{R}}}\left(\frac{2\left(L_{\mathrm{R}}-1\right)}{L_{\mathrm{R}}} Q\left(\sqrt{\frac{6 \rho_{k}}{L_{\mathrm{R}}^{2}-1}}\right)\right)
$$

where $\mathcal{Q}(\cdot)$ is the Gaussian-Q function, and the approximation in (35) comes from the employment of $\log _{2}^{-1}\left(L_{\mathrm{R}}\right)$ for converting the symbol error rate to the BER.

When the MIMO channel is i.i.d. complex Gaussian with zero mean, the instantaneous SNR $\rho_{k}$ is chi-square distributed with $\rho_{k} \sim \chi^{2}\left(2 D_{\text {Rснм }}\right)$ [18]. Following the procedure in [15], [40], the average BER over fading channels is

$$
\begin{aligned}
& \overline{\mathcal{P}}_{e, \mathrm{ASK}}^{(0)}=\underset{\rho_{k}}{\mathbb{E}}\left(\mathcal{P}_{e, \mathrm{ASK}}\left(\rho_{k}\right)\right) \\
& \approx \frac{1}{\log _{2} L_{\mathrm{R}}}\left(\frac{L_{\mathrm{R}}-1}{L_{\mathrm{R}}}-\frac{2\left(L_{\mathrm{R}}-1\right)}{L_{\mathrm{R}}} \sqrt{\frac{3 \gamma_{o}}{\pi\left(L_{\mathrm{R}}^{2}-1\right)}}\right. \\
& \left.\times \frac{\Gamma\left(D_{\text {Rснм }}+\frac{1}{2}\right)}{\Gamma\left(D_{\text {Rснм }}\right)}{ }_{2} F_{1}\left(\frac{1}{2}, D_{\text {Rснм }}+\frac{1}{2} ; \frac{3}{2} ; \frac{-3 \gamma_{o}}{L_{\mathrm{R}}^{2}-1}\right)\right)
\end{aligned}
$$

where ${ }_{2} F_{1}(, ; ;)$ is the hypergeometric function, $\mathbb{E}(\cdot)$ the expectation, and $\mathbb{E}\left(\rho_{k}\right)=\gamma_{o}$ assuming the power allocation suggested by (30). Similarly, the average BER for $L_{\mathrm{C}}$-QAM symbol within $\breve{\mathbf{z}}$ can be derived following the procedure in [41]

$$
\begin{aligned}
\overline{\mathcal{P}}_{e, \mathrm{QAM}}^{(0)} & \approx \frac{1}{\log _{2}\left(\sqrt{L_{\mathrm{C}}}\right)}\left(\frac{\sqrt{L_{\mathrm{C}}}-1}{\sqrt{L_{\mathrm{C}}}}-\frac{2\left(\sqrt{L_{\mathrm{C}}}-1\right)}{\sqrt{L_{\mathrm{C}}}} \sqrt{\frac{3 \gamma_{o}}{\pi\left(L_{\mathrm{C}}-1\right)}}\right. \\
& \times \frac{\Gamma\left(D_{\mathrm{RCHM}}+1\right)}{\Gamma\left(D_{\mathrm{RCHM}}+\frac{1}{2}\right)}{ }_{2} F_{1}\left(\frac{1}{2}, D_{\mathrm{RCHM}}+1 ; \frac{3}{2} ; \frac{-3 \gamma_{o}}{L_{\mathrm{C}}-1}\right)(\beta 8)
\end{aligned}
$$

Plugging (37)-(38) into (35) gives the average BER for the initial state of ZF-SIC.

To facilitate our BER analysis for the iterative process, we propose a state transition diagram; as shown in Fig. 3. Basically, the notation $S_{k}, k \in[0, K]$, denotes the transition states, where $k$ ASK-QAM sequences have been successfully reconstructed and subtracted. For instance, $S_{0}$ is the initial state of the ZF-SIC receiver, where no sequence is subtracted yet, and $S_{K}$ is which all sequences have been successfully reconstructed. The notation $E_{k}, k \in[0, K-1]$, denotes the error state related to the corresponding state $S_{k}$. For instance, $E_{0}$ is the error state for the initial state of the ZF-SIC process, and the state $E_{K-1}$ represents the error state for the last residual sequence. According to the algorithm description s1)-s4), the iterative process starts from $S_{0}$ and can terminate at any other states.

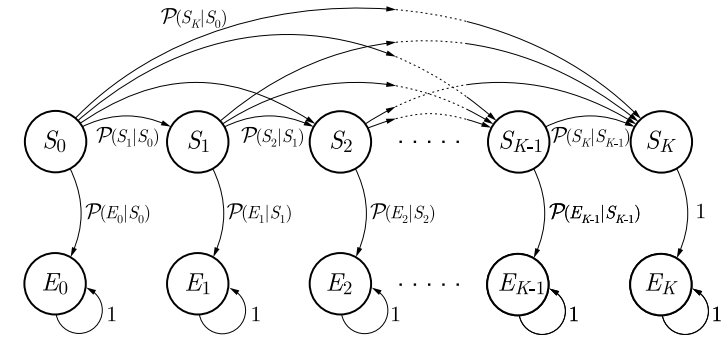

Fig. 3. State transition diagram for the BER analysis considering ZF-SIC receiver.

According to the state transition diagram, the overall average BER for the ZF-SIC process is given by

$$
\overline{\mathcal{P}}_{e, \mathrm{AQAM}}=\sum_{k=1}^{K} \mathcal{P}\left(E_{k}\right) \cdot \overline{\mathcal{P}}_{e, \mathrm{AQAM}}^{(k)} f(k)
$$

where $\overline{\mathcal{P}}_{e, \mathrm{AQAM}}^{(k)} f(k)$ is the average BER at $E_{k}, \overline{\mathcal{P}}_{e, \mathrm{AQAM}}^{(k)}$ the average BER at $S_{k}{ }^{4}$, and $f(k)$ is defined by

$$
f(k)= \begin{cases}(K-k) / K, & k \neq K \\ 1, & k=K\end{cases}
$$

Note that the BER forms (35), (37)-(38) also hold for $\overline{\mathcal{P}}_{e, \mathrm{AQAM}}^{(k)}$ with changes applying to the diversity parameter $D_{\mathrm{RCHM}}$, which should now be labeled with the superscript $(\cdot)^{(k)}$, i.e., $D_{\text {RCHM }}^{(k)}$. This is because successfully reconstructed sequences have been removed from the input waveform, and such changes the parameter $K$ to $(K-k)$. We notice that, after removing $k$ sequences, spatial blocks might not have identical realto-complex symbol ratio, and thus $D_{\mathrm{RCHM}}^{(k)}$ can vary between spatial blocks. This phenomenon largely complicates the BER analysis. Nevertheless, we can simplify the analysis using

$$
D_{\mathrm{RCHM}}^{(k)}=M-\frac{K+K_{\mathrm{C}}-1-\frac{k}{K}\left(K+K_{\mathrm{C}}\right)}{2}
$$

which assumes even reduction of real and complex symbols for every spatial block. Our simulation results in Section V confirms this assumption to be sufficiently accurate. In addition, the term $\gamma_{o}$ should be replaced by: $\gamma_{o}^{(k)}=\gamma_{o}(K-k) / K$, which reflects the signal power reduction due to the sequence subtraction. In order to prevent possible over-approximation throughout the above BER analysis, we let

$$
\overline{\mathcal{P}}_{e, \mathrm{AQAM}}^{(k)}=\max \left(\overline{\mathcal{P}}_{e, \mathrm{AQAM}}^{(k)}, \overline{\mathcal{P}}_{e, \mathrm{ML}}\right)
$$

where $\overline{\mathcal{P}}_{e, \mathrm{ML}}$ is the ML lower bound for the average BER. According to [18], it is obtained by applying the maximal diversity-order $D_{\mathrm{RCHM}}=M$ in (35), (37)-(38).

Now, our interest moves to the term $\mathcal{P}\left(E_{k}\right)$ in (39). As illustrated in Fig. 3, it is given by

$$
\mathcal{P}\left(E_{k}\right)=\mathcal{P}\left(E_{k} \mid S_{k}\right) \mathcal{P}\left(S_{k}\right)
$$

where $\mathcal{P}\left(E_{k} \mid S_{k}\right)$ is the probability of $E_{k}$ conditioned on $S_{k}$, and $\mathcal{P}\left(S_{k}\right)$ the probability of reaching the state $S_{k}$, which can

\footnotetext{
${ }^{4}$ The BER at $S_{k}$ is the number of error bits to all the bits within the $(K-k)$ remaining sequences, and the BER at $E_{k}$ is the number of error bits to the bits within all the $K$ sequences.
} 
be expressed in a recursive form

$$
\mathcal{P}\left(S_{k}\right)=\sum_{j=0}^{k-1} \mathcal{P}\left(S_{k} \mid S_{j}\right) \mathcal{P}\left(S_{j}\right)
$$

with $\mathcal{P}\left(S_{0}\right)=1$. The conditional probabilities $\mathcal{P}\left(E_{k} \mid S_{k}\right)$ and $\mathcal{P}\left(S_{k} \mid S_{j}\right)$ are related to the block error rate (BLER) on the corresponding states, which vary with respect to MIMO channel realizations. Hence, we utilize the approximate BLER on state $S_{j}$ [42]

$$
\mathrm{BLER}_{j} \approx 1-\left(1-\overline{\mathcal{P}}_{e, \mathrm{AQAM}}^{(j)}\right)^{N_{b}}
$$

where $N_{b}=N_{\mathrm{R}} \log _{2}\left(L_{\mathrm{R}}\right)+N_{\mathrm{C}} \log _{2}\left(L_{\mathrm{C}}\right)$ is the number of bits per sequence. Section V will confirm (45) to be sufficiently accurate. Finally, the conditional probability $\mathcal{P}\left(S_{k} \mid S_{j}\right)$ is equivalent to the probability of all possible combinations of $(k-j)$ out of $(K-j)$ ASK-QAM sequences that can be successfully reconstructed. Hence, it is given by

$$
\mathcal{P}\left(S_{k} \mid S_{j}\right)=\left(\begin{array}{c}
K-j \\
k-j
\end{array}\right)\left(1-\mathrm{BLER}_{j}\right)^{k-j} \mathrm{BLER}_{j}^{K-k}
$$

and accordingly we have

$$
\mathcal{P}\left(E_{k} \mid S_{k}\right)=1-\sum_{k=j+1}^{K} \mathcal{P}\left(S_{k} \mid S_{j}\right)=\mathrm{BLER}_{k}^{K-k} .
$$

Such concludes the average BER analysis of the ZF-SIC receiver for ASK-QAM modulated MU-MIMO systems.

\section{Receiver Complexity}

The computational complexity of ZF-SIC receiver mainly comes from the channel matrix inverse and the iterative interference cancellation process. Considering the $i^{\text {th }}$ iteration, there are $Q_{i}$ different patterns for the spatial blocks, and the channel matrix $\overline{\mathbf{H}}$ in (19) has $Q_{i}$ different corresponding forms. Then, the overall computational cost for channel matrix inverse is

$$
\sum_{i=0}^{I} \mathcal{O}\left(Q_{i} K_{i}^{2} M\right)
$$

where $I$ denotes the number of iterations, and $K_{i}$ the number of residual sequences for the $i^{\text {th }}$ iteration.

Comparing with the linear ZF receiver, the ZF-SIC receiver for RCHM-MIMO introduces extra computational complexity due to the parameters $I$ and $Q$. Section $\mathrm{V}$ will show that the $\mathrm{ZF}$-SIC receiver features fast convergence (i.e., $I \ll K$ ), and we can carefully design the pattern of spatial block so as to make $Q \ll K$.

\section{Simulation Results and Discussion}

\section{A. Configuration of Key Parameters for RCHM-MIMO}

The primary objective of computer simulations is to evaluate RCHM-MIMO uplink communications with emphasis on the use of equal service antennas and UTs $(M=K)$; as this is the case that motivates the RCHM-MIMO design. The modulation schemes employed for RCHM are $L_{R}-\mathrm{ASK}$ and $L_{\mathrm{C}}$-QAM. According to the theoretical work in Section III, the real-to-complex symbol ratio is set to: $\alpha=\beta=1$, which is optimized for higher data-rate such as $4-6 \mathrm{bit} / \mathrm{s} / \mathrm{Hz} / \mathrm{UT}$ (see Fig. 2). Moreover, the equal use of real and complex modulations can largely simplify the transceiver design. In our simulation examples, UTs have binary option for their signal pattern transmissions, which remain unchanged during each temporal block: one follows the pattern [ASK, QAM, ..., ASK, QAM], and the other follows [QAM, ASK, ..., QAM, ASK]. The network will inform UTs regarding their pattern with 1-bit signalling, and this is already the minimal signalling overhead fulfilling the design criterion C3). When signal patterns are equally assigned to UTs, the design criteria $\mathrm{C} 1$ )-C2) are also satisfied. It is worth noting that the proposed signal pattern is not the only one that can fulfill the design criteria, and our simulation results show that this proposed signal pattern is already near-optimal.

\section{B. Simulations and Performance Evaluation}

The key metric utilized for performance evaluation is the average BER (or average BLER in the case of FEC) as a function of average received information bit-energy to noise ratio per receive antenna (i.e., $E_{b} / N_{0}$ ). At the transmitter, equal power allocation is applied at the bit level, which is in line with the power allocation strategy suggested by (30). The channel model of small-scale fading is the Extended Pedestrian-A (EPA) specified by $3^{\text {rd }}$ Generation Partnership Project (3GPP) [43], with each tap independently generated according to the complex Gaussian distribution. Despite the channel frequency selectivity, our investigation is conducted only on one of frequency flat sub-channels in an OFDM system; as this helps to have a better demonstration of the spatial diversity-multiplexing gain as well as the technical implication of RCHM-MIMO. Specifically, our computer simulations include five experiments.

Experiment 1: The aim of this experiment is to examine the average BER performance of RCHM-MIMO at the link level, where each UT-to-receiver link has the identical large-scale path loss, and the MIMO channel matrix $\mathbf{H}$ is normalized; such is the commonly utilized configuration for MU-MIMO link-level simulations (e.g. [4], [6], [9], [11], [15] and many others.) In this experiment, we consider every data sequence having 64 uncoded bits, and 8-bit CRC with the polynomial '0x97' (see [44]) is employed for error detection at the sequence level. The average bit/symbol is: 3 (4-ASK/16-QAM), 4 (4-ASK/64-QAM) or 6 (16-ASK/256-QAM), respectively. We have tested all possible combinations of $L_{\mathrm{R}}$-ASK and $L_{\mathrm{C}^{-}}$ QAM for each considered bit/symbol and found these the best combinations in terms of the performance. Hence, they will be utilized throughout Section V.

In Fig. 4, we compare simulation results with the theoretical BER (39) and the ML lower bound for 20-by-20 RCHMMIMO. It is observed that the theoretical BERs are very close to their corresponding simulations results $(<0.5 \mathrm{~dB})$, and in general the theoretical BERs are lower than their corresponding simulation results. This is mainly due to the use of approximated BLER in (45). Moreover, it is observed that the difference between simulation results and the ML lower bound is also very close $(<1 \mathrm{~dB})$. This clearly indicates that the ZF-SIC receiver is near-optimal for RCHM-MIMO. 


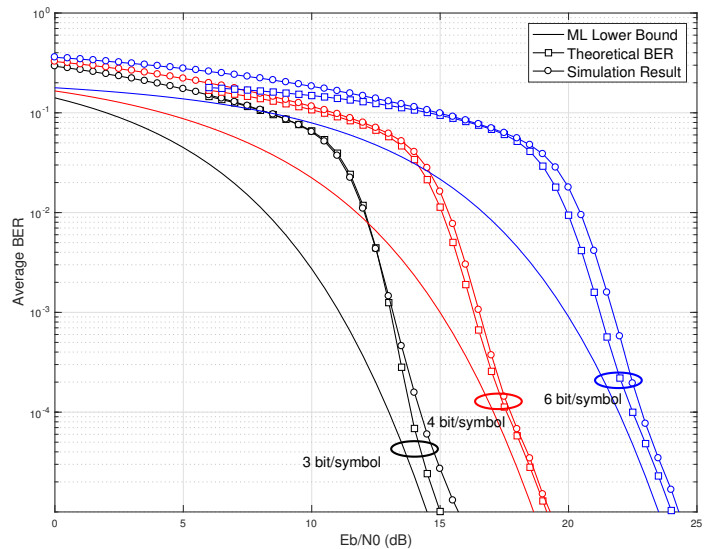

Fig. 4. average BER as a function of $E_{b} / N_{0}$ for uncoded 20-by-20 RCHMMIMO system.

Fig. 5 demonstrates the average BER performances for various sizes of RCHM-MIMO. It is observed that, at high SNRs (e.g., $E_{b} / N_{0}>10 \mathrm{~dB}$ in the case of $3 \mathrm{bit} / \mathrm{symbol}$ ), the BER performances improve with the increasing size of RCHM-MIMO. It means that the ZF-SIC receiver can efficiently exploit the spatial diversity gain of a larger MU-MIMO system. Here, we stress that most of low-complexity MUMIMO receivers can enjoy the spatial diversity gain only when they have the knowledge of SNR (or SINR in the presence of interferences, e.g. [6], [12]), which is not needed at all in the RCHM-MIMO system. It is also observed that BER curves with the same bit/symbol are very close to each other at low SNRs. This phenomenon coincides with the well-known conclusion that diversity gain appears only at high SNRs [20].

Another remarkable phenomenon is: when the size of RCHM-MIMO gets sufficiently large (e.g. $M=K=64$ or 128), the average BER is very close to the BER of single-antenna point-to-point (i.e., SISO) communication in the AWGN channel. The difference between them is less than $0.8 \mathrm{~dB}$ in SNR. This is another evidence that the ZF-SIC receiver is near optimal for RCHM-MIMO. It is perhaps worth noting that the small gap between the BERs of 128-by-128 RCHM-MIMO and SISO AWGN is mainly due to the CRC overhead, which results in more than $0.5 \mathrm{~dB}$ loss in SNR.

In Fig. 6, we compare the average BER performances between RCHM-MIMO and conventional MU-MIMO. Due to the limited space, we only use the average spectral efficiency of $4 \mathrm{bit} / \mathrm{symbol}$ as an example, where correspondingly 16QAM is considered for conventional MU-MIMO systems. Our simulation results are coincide with the well-known conclusion in the literature that conventional MU-MIMO is not a scalable technology. Both the ZF-SIC and LR-ZF receivers are too sub-optimal in MU-MIMO systems $(10-15 \mathrm{~dB}$ away from the RCHM-MIMO result), and their performances get worse with the increase of MIMO size. We also compare RCHMMIMO with 16-QAM SISO in an AWGN channel; the latter serves as the BER lower bound for conventional MU-MIMO systems. We can see that the difference between two systems is around $5 \mathrm{~dB}$ in SNR, which is mainly due to the multiplexing

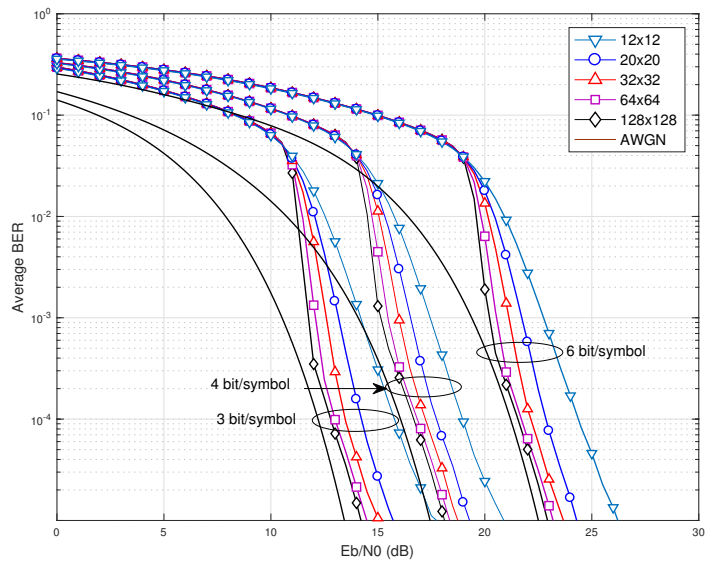

Fig. 5. average BER as a function of $E_{b} / N_{0}$ for uncoded RCHM-MIMO system of different size.

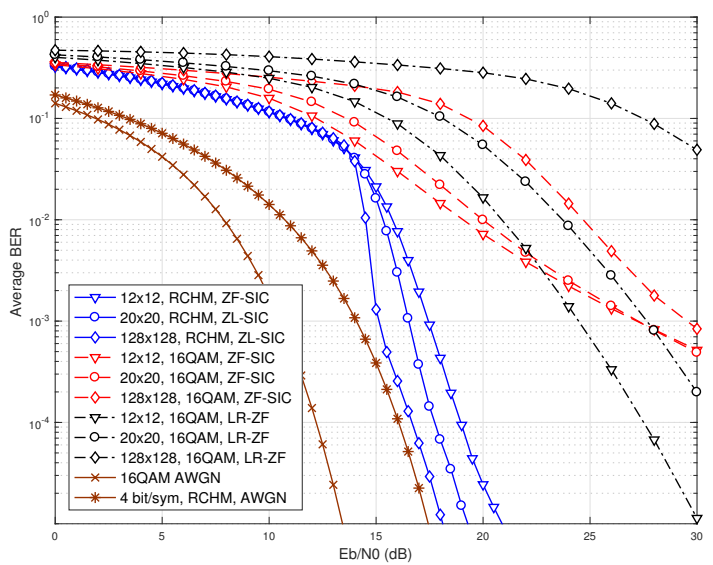

Fig. 6. Performance comparison between RCHM-MIMO and baselines. For baseline techniques, 16-QAM is utilized as an example; and correspondingly the RCHM technique adopts 4 bits/symbol.

inefficiency of using $L_{\mathrm{R}}$-ASK modulation. Nevertheless, to the best of our knowledge, such is the best performance that a low-complexity approach can offer.

Experiment 2: The objective of this experiment is to demonstrate the convergence of ZF-SIC receiver, of which the impact on the receiver complexity is also discussed.

Fig. 7 shows the average number of iterations required for the ZF-SIC receiver to reach the end of the iterative process. Generally, RCHM-MIMO of a larger size requires more iterations. For instance in the case of 4 bits/symbol, the peak of average iterations is around 4.5 for 20-by-20 RCHMMIMO, and increases to 6 for 32-by-32 MIMO or 13 for 128by-128 RCHM-MIMO. Such a difference occurs only at the peak or a small range around (e.g., $E_{b} / N_{0} \in[13,16] \mathrm{dB}$ in the case of 4 bits/symbol). At higher SNRs, the average number of iterations decreases considerably.

To explain this phenomenon, we study Fig. 5 and Fig. 7 together. It can be observed that the peak of iteration numbers appears at the SNR range where the spatial diversity gain 


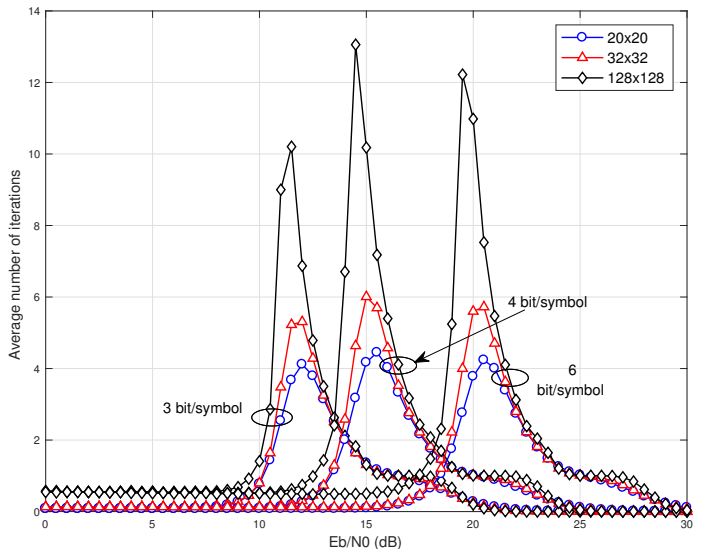

Fig. 7. Average number of iterations of the ZF-SIC receiver vs. $E_{b} / N_{0}$ for various RCHM-MIMO sizes

emerges. For smaller SNRs outside this range, MU-MIMO systems of different sizes have almost identical and large BERs. In this case, it is hard to have a single RCHM sequence to be successfully recovered at the initial widelylinear processing state, i.e., $\mathcal{P}\left(E_{0}\right)$ is close to 1 (see Fig. 3); and thus the detection process terminates after the initial stage with a large probability. For larger SNRs outside the peak range, the BERs decrease quickly to a small value (e.g., $0.1 \%$ or below) thanks to the large spatial diversity gain. In this case, for most of channel realizations, all ASK-QAM sequences are successfully recovered at the initial ZF channel equalization stage or the first two iterations, i.e., the receiver only needs a small number of iterations to reach the state $S_{K}$ (see Fig. 3) with a large probability.

Moreover, we take 20-by-20 MU-MIMO as an example, and plot in Fig. 8 the average BER with respect to the number of iterations. Note that this result reflects the statistical behaviour of convergence in fading channels. It is observed that the average BER performance is saturated after three iterations, and further iteration does not considerably improve the BER performance. The same conclusion can be drawn for other sizes of RCHM-MIMO. Then, the computational complexity of ZF-SIC receiver is now approximately equal to (derived from (48) with $I=3$ ): $\sum_{i=0}^{3} \mathcal{O}\left(Q_{i} K_{i}^{2} M\right)$.

Experiment 3: This experiment aims to demonstrate the impact of using different real-to-complex ratio (i.e., $\alpha$ ) on the performance of RCHM-MIMO. The system is $24 \times 24$ RCHM-MIMO without FEC. The modulation scheme is the hybrid of 16-ASK and 256-QAM with the real-to-complex ratio: $\alpha=2 / 1,1 / 1,1 / 2,1 / 3$. Fig. 9 shows the throughput vs. $E_{b} / N_{0}$. It is observed that the peak throughput is inversely proportional to $\alpha$. On the other hand, the cases with larger $\alpha$ offers better throughput at relatively lower SNRs. These results are fully in line with the spatial diversity-multiplexing trade-off discussed in Section III-B.

Experiment 4: This experiment aims to demonstrate the performance of FEC-coded RCHM-MIMO systems as well as the impact of channel estimation errors. In this experiment, every block has 640 information bits. The CRC code is CRC-

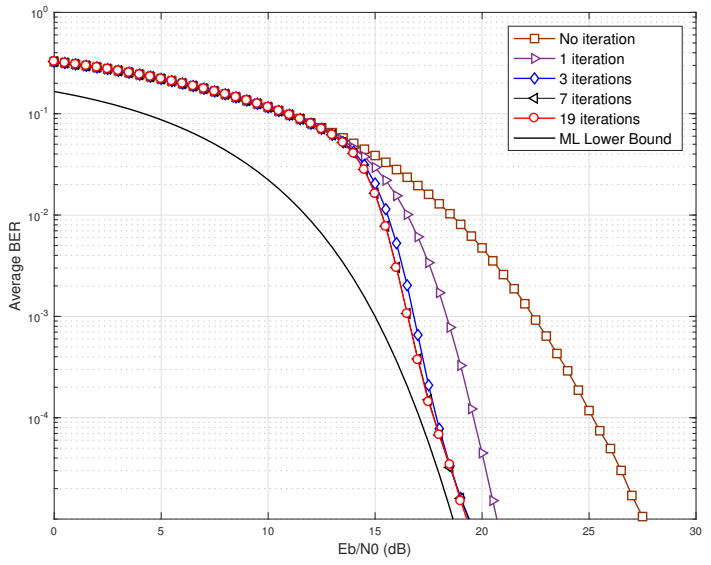

Fig. 8. average BER as a function of SNR for uncoded 20-by-20 RCHMMIMO system with respect to the maximal number of iterations. The spectral efficiency is $4 \mathrm{bit} / \mathrm{symbol}$.

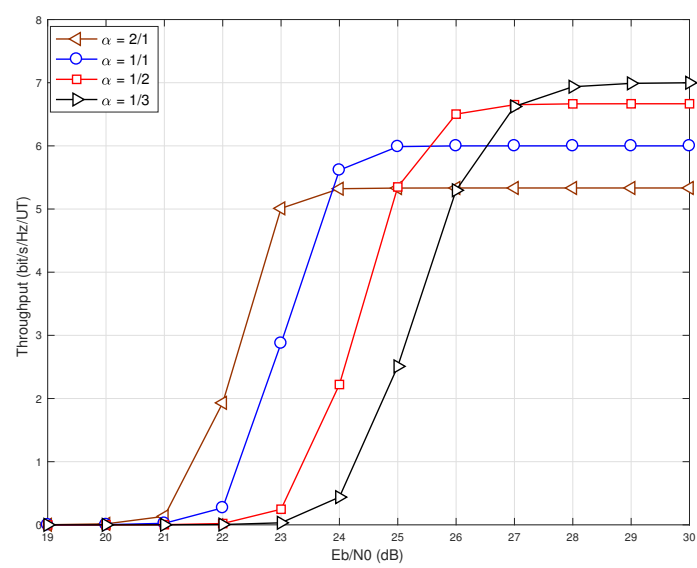

Fig. 9. Throughput in bit/s/Hz/UT for 24-by-24 RCHM-MIMO with differen real-to-complex ratios: $\alpha=2 / 1,1 / 1,1 / 2,1 / 3$. The modulations are hybrid of $16-\mathrm{ASK}$ and 256-QAM.

24 specified in the LTE standard [45], and the FEC code is turbo code (TC) with the code-rate of $1 / 3$ or $5 / 6$. The rate$1 / 3 \mathrm{TC}$ is constructed according to the structure recommended by LTE, and the rate- $5 / 6 \mathrm{TC}$ is constructed using the ratematch method also specified in the LTE standard [45]. The demodulator utilizes the soft de-mapping approach proposed in [46] to form soft decision with approximate log-likelihood ratio (LLR), of which the output is then fed into the turbo decoder. By this means, the SNR/SINR knowledge is not needed in the demodulation and decoding process.

Fig. 10 illustrates the average-BLER as a function of $E_{b} / N_{0}$. In contrast with the uncoded results in Fig. 4, we can observe $6 \mathrm{~dB}$ or more gain with rate- $1 / 3 \mathrm{TC}$ and $2.5 \mathrm{~dB}$ or more gain with rate- $5 / 6 \mathrm{TC}$. It is also observed that the BLER curves are very steep, and very low BLER (e.g. 1\% or smaller) can be achieved at moderate SNRs (e.g. $E_{b} / N_{0}$ $\in[5,20] \mathrm{dB})$.

Fig. 10 also illustrated the average-BLER performance when the least-squares (LS) channel estimation is employed 


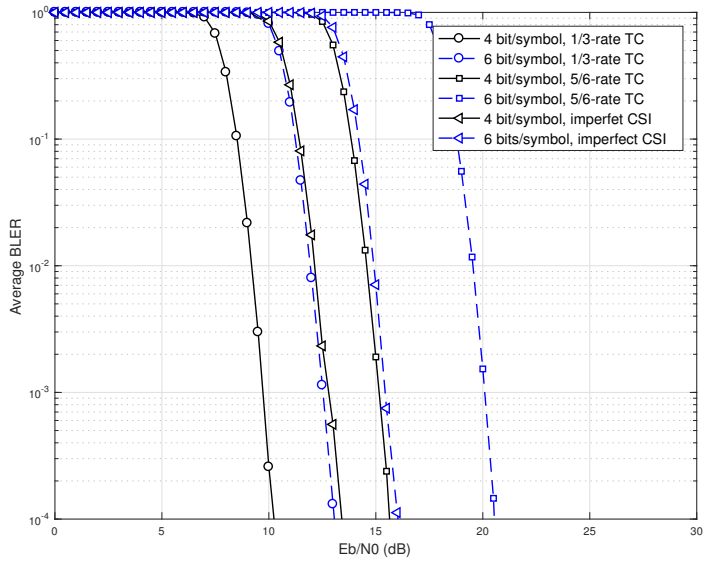

Fig. 10. Average-BLER of rate-1/3 and rate-5/6 turbo coded RCHM-MIMO in 20-by-20 MU-MIMO systems with perfect or imperfect CSI.

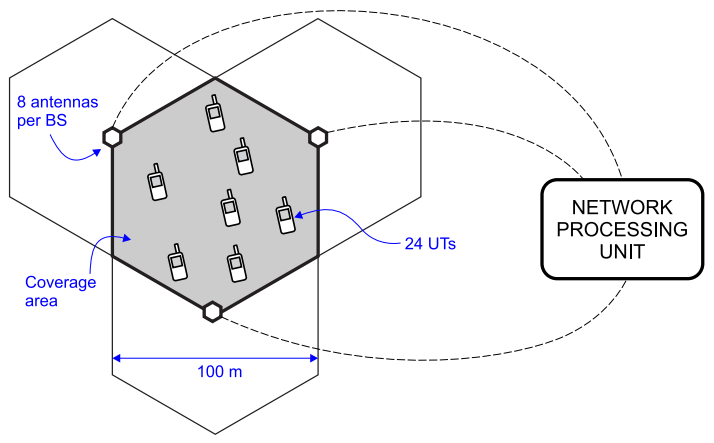

Fig. 11. Three cooperating BSs in an edge-excited cell setup.

to obtain the channel state information (CSI). According to the information-theoretic result in [47], [48], we employ 20 pilots per UT for the LS channel estimation. Pilots and payload symbols have identical power. By this means, the accumulated variance of channel estimation errors at each receive antenna is equal to the noise variance. In this case, the impact of channel estimation errors is theoretically equivalent to $3 \mathrm{~dB}$ degradation in SNR. This theoretical result is well confirmed by simulation results depicted in Fig. 10.

Experiment 5: The aim of this experiment is to compare the average-BLER performances of RCHM-MIMO and conventional MU-MIMO considering an edge-excited cell [49], where three cooperating BSs with a 100 meter inter-BS distance, send their baseband signals to a network processing unit that performs the joint detection, assuming perfect front-hauls; as shown in Fig. 11. Each BS contains an linear array with 8 semi-directional antennas $(M=3 \times 8=24)$ with a $60^{\circ}$ aperture, oriented towards the center of the cell. Co-located receive antennas at each $\mathrm{BS}$ are correlated with the correlation coefficient of 0.6 , and an exponential correlation model [50] is used in our simulations. Receive antennas belonging to different BSs are uncorrelated. The cell serves 24 UTs $(K=24)$ that are uniformly distributed within the coverage area. The channel model incorporates both the small-scale fading and large-scale path loss with shadowing. The small-scale fading uses the same channel model as used in Experiments 1-4 with

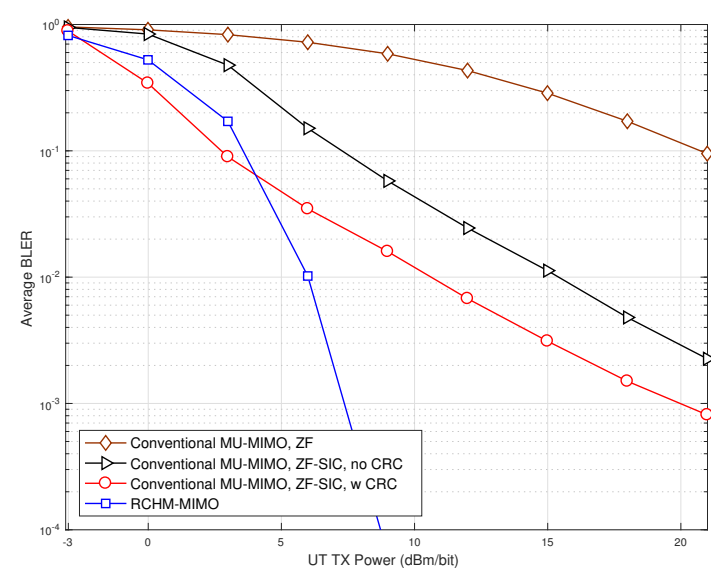

Fig. 12. Average BLER vs. UT transmit power $(\mathrm{dBm} / \mathrm{bit})$ with the cellexcited setup.
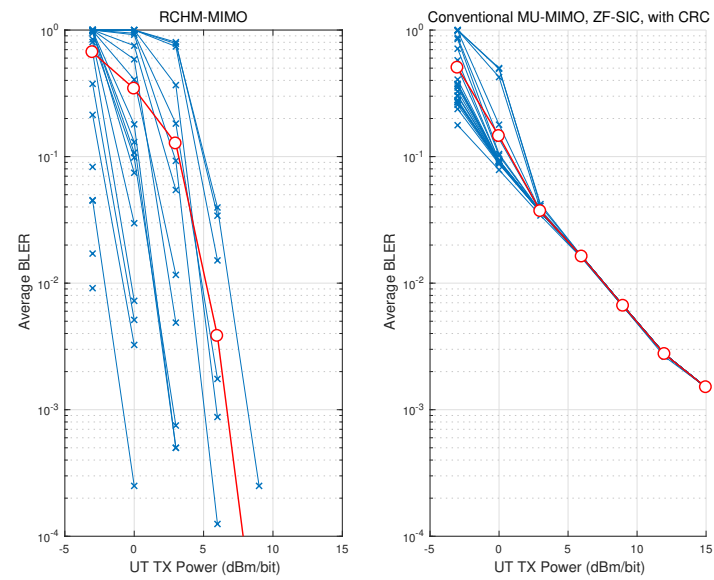

Fig. 13. The average BLER of each individual user for RCHM-MIMO (left) and conventional MU-MIMO (right).

the additional consideration of antenna correlation. The largescale effects are incorporated in the matrix $\mathbf{A}$ described in (1), with each element of $\mathbf{A}$ to be distributed according to the UMi open square, with the path-loss exponent to be 2.8 and a shadowing standard deviation of $8.3 \mathrm{~dB}$; as described in [51] for $5 \mathrm{G}$ scenarios. Other key parameters include: 6 bit/symbol ASK-QAM hybrid modulation; rate-5/6 TC; UT height of $1.65 \mathrm{~m}$ and $\mathrm{BS}$ height of $10 \mathrm{~m} ; 2 \mathrm{GHz}$ carrier frequency with 20 $\mathrm{MHz}$ signal bandwidth; $-174 \mathrm{dBm} / \mathrm{Hz}$ noise spectral density; 640 uncoded bits per block, and CRC-24.

Fig. 12 shows that RCHM-MIMO generally outperforms the conventional MU-MIMO in terms of the transmit-power efficiency. The only exceptional case is at lower transmitpower regime $(<3 \mathrm{dBm} / \mathrm{bit})$, where conventional MU-MIMO with CRC-enhanced ZF-SIC receiver outperforms RCHMMIMO. To understand this interesting phenomenon, we plot in Fig. 13 the BLER results for each individual user. It is observed that: UTs in the conventional MU-MIMO system have relatively close BLERs, while in RCHM-MIMO, UT's BLER is very location dependent, i.e., UTs close to a BS have 
much better performance than those located at the center of the edge-excited cell. This clearly indicates that UTs in the conventional MU-MIMO have the performance limited by their mutual interference, and those in RCHM-MIMO have their performances limited by the noise. In other words, RCHMMIMO has much stronger capability for mutual interference cancellation, and the BLER at lower transmit-power regime can be improved through uplink power control.

\section{CONCLUSION}

This paper has introduced a novel RCHM-MIMO technology that can significantly improve the scalability of MUMIMO uplink communications particularly for fully-loaded or heavily-loaded systems. The performance of RCHM-MIMO has been carefully evaluated through the theoretical analysis of the system outage probability, average BER or BLER, as well as extensive computer simulations considering a network with single or multiple BSs. The RCHM pattern has been carefully designed in terms of the minimized outage probability, transmit-power efficiency, or minimized signaling overhead. It has been shown that the ZF-SIC receiver is near optimal for RCHM-MIMO systems, and the RCHM-MIMO system as a whole can offer near-optimal MU-MIMO uplink communications with the receiver complexity comparable with the linear $\mathrm{ZF}$ algorithm.

\section{REFERENCES}

[1] T. Marzetta, "Noncooperative cellular wireless with unlimited number of base station antennas," IEEE Trans. Wireless Commun., vol. 9, pp. 3590-3600, Nov. 2010.

[2] E. G. Larsson, O. Edfors, F. Tufvesson, and T. L. Marzetta, "Massive MIMO for next generation wireless systems," IEEE Commun. Mag., no. 2, pp. 186-195, Feb. 2014.

[3] E. Larsson, "MIMO detection methods: How they work [lecture notes]," IEEE Signal Process. Mag., vol. 26, pp. 91-95, May 2009.

[4] Y. Ma, A. Yamani, N. Yi, and R. Tafazolli, "Low-complexity MU-MIMO nonlinear precoding using degree-2 sparse vector perturbation," IEEE $J$. Sel. Areas Commun., vol. 34, no. 3, pp. 497-509, Mar. 2016.

[5] D. Wubben, D. Seethaler, J. Jalden, and G. Matz, "Lattice reduction," IEEE Signal Process. Mag., vol. 28, pp. 70-91, May 2011.

[6] Q. Zhou and X. Ma, "Element-based lattice reduction algorithms for large MIMO detection," IEEE J. Sel. Areas Commun., vol. 31, no. 2 , pp. 274-286, Feb. 2013

[7] P. Wolniansky, G. Foschini, G. Golden, and R. Valenzuela, "V-BLAST: An architecture for realizing very high data rates over the rich-scattering wireless channel," in Proc. URSI Int. Symp. Signals, Syst., and Electronics Conf., Sep. 1998, pp. 295-300.

[8] Z.-Q. Luo, W.-K. Ma, A.-C. So, Y. Ye, and S. Zhang, "Semidefinite relaxation of quadratic optimization problems," IEEE Signal Process. Mag., vol. 27, no. 3, pp. 20-34, May 2010.

[9] K. Vardhan, S. Mohammed, A. Chockalingam, and B. Rajan, "A lowcomplexity detector for large MIMO systems and multicarrier CDMA systems," IEEE J. Sel. Areas Commun., vol. 26, pp. 473-485, Apr. 2008.

[10] H. Zhao, H. Long, and W. Wang, "Tabu search detection for MIMO systems," in Proc. IEEE 18th Int. Symp. Personal Indoor and Mobile Radio Commun. (PIMRC), Sep. 2007, pp. 1-5.

[11] A. Chockalingam and B. Sundar Rajan, Large MIMO Systems. Cambridge University Press, 2014

[12] P. Som, T. Datta, A. Chockalingam, and B. Rajan, "Improved largeMIMO detection based on damped belief propagation," in IEEE Inform. Theory Workshop (ITW), Jan. 2010, pp. 1-5.

[13] Y.-C. Liang, S. Sun, and C. K. Ho, "Block-iterative generalized decision feedback equalizers for large MIMO systems: Algorithm design and asymptotic performance analysis," IEEE Trans. Signal Process., vol. 54, no. 6, pp. 2035-2048, Jun. 2006.

[14] F. Rusek, D. Persson, B. K. Lau, E. Larsson, T. Marzetta, O. Edfors, and F. Tufvesson, "Scaling up MIMO: Opportunities and challenges with very large arrays," IEEE Signal Process. Mag., vol. 30, no. 1, pp. 40-60, Jan. 2013.
[15] J. De Luna Ducoing, N. Yi, Y. Ma, and R. Tafazolli, "Using rea constellations in fully- and over-loaded large MU-MIMO systems with simple detection," IEEE Wireless Commun. Lett., vol. 5, no. 1, pp. 92 95, Feb. 2016

[16] Y. Li, J. Winters, and N. Sollenberger, "MIMO-OFDM for wireless communications: Signal detection with enhanced channel estimation,' IEEE Trans. Commun., vol. 50, no. 9, pp. 1471-1477, Sep. 2002.

[17] X. Wang, P. Zhu, and M. Chen, "Thresholded interference cancellation algorithm for the LTE uplink multiuser MIMO," in Proc. IEEE Global Commun. (GLOBECOM) Conf., Nov. 2008, pp. 1-5.

[18] M. Rupp, C. Mecklenbrauker, and G. Gritsch, "High diversity with simple space time block-codes and linear receivers," in Proc. IEEE Global Telecommun. (GLOBECOM) Conf., Dec. 2003, pp. 302-306.

[19] A. Hedayat and A. Nosratinia, "Outage and diversity of linear receivers in flat-fading MIMO channels," IEEE Trans. Signal Process., vol. 55, no. 12 , pp. 5868-5873, Dec. 2007.

[20] D. Tse and P. Viswanath, Fundamentals of wireless communication. Cambridge University Press, 2005.

[21] B. Picinbono, "On circularity," IEEE Trans. Signal Process., vol. 42, no. 12 , pp. 3473-3482, Dec 1994

[22] B. Picinbono and P. Chevalier, "Widely linear estimation with complex data," IEEE Trans. Signal Process., vol. 43, no. 8, pp. 2030-2033, Aug. 1995.

[23] C. Pietsch, S. Sand, W. Teich, and J. Lindner, "Modeling and performance evaluation of multiuser MIMO systems using real-valued matrices," IEEE J. Sel. Areas Commun., vol. 21, pp. 744-753, Jun. 2003.

[24] S. Sfar, R. D. Murch, and K. B. Letaief, "Layered space-time multiuser detection over wireless uplink systems," IEEE Trans. Wireless Commun., vol. 2, no. 4, pp. 653-668, Jul. 2003.

[25] S. Buzzi, M. Lops, and S. Sardellitti, "Widely linear reception strategies for layered space-time wireless communications," IEEE Trans. Signal Process., vol. 54, pp. 2252-2262, Jun. 2006.

[26] P. Chevalier and F. Pipon, "New insights into optimal widely linear array receivers for the demodulation of BPSK, MSK, and GMSK signals corrupted by noncircular interferences-Application to SAIC," IEEE Trans. Signal Process., vol. 54, pp. 870-883, Mar. 2006.

[27] P. Chevalier and F. Dupuy, "Widely linear Alamouti receiver for the reception of real-valued constellations corrupted by interferences-The Alamouti-SAIC/MAIC concept," IEEE Trans. Signal Process., vol. 59, pp. 3339-3354, Jul. 2011.

[28] R. Meyer, W. Gerstacker, R. Schober, and J. Huber, "A single antenna interference cancellation algorithm for increased GSM capacity," IEEE Trans. Wireless Commun., vol. 5, pp. 1616-1621, Jul. 2006.

[29] R. Fischer and C. Windpassinger, "Real versus complex-valued equalisation in V-BLAST systems," IET Electron. Lett., vol. 39, pp. 470-471, Mar. 2003.

[30] M. Witzke, "Linear and widely linear filtering applied to iterative detection of generalized MIMO signals," in Annales des télécommunications, vol. 60,2005 , pp. 147-168.

[31] W. H. Gerstacker, F. Obernosterer, R. Schober, A. T. Lehmann, A. Lampe, and P. Gunreben, "Equalization concepts for Alamouti's space-time block code," IEEE Trans. Commun., vol. 52, no. 7, pp. 11781190, Jul. 2004

[32] Y. Zeng, C. M. Yetis, E. Gunawan, Y. L. Guan, and R. Zhang, "Transmit optimization with improper Gaussian signaling for interference channels," IEEE Trans. Signal Process., vol. 61, no. 11, pp. 2899-2913, Jun. 2013.

[33] Y. Zeng, R. Zhang, E. Gunawan, and Y. L. Guan, "Optimized transmission with improper Gaussian signaling in the K-user MISO interference channel," IEEE Trans. Wireless Commun., vol. 12, no. 12, pp. 6303 6313, Dec. 2013.

[34] R. Mesleh, H. Haas, S. Sinanovic, C. W. Ahn, and S. Yun, "Spatial modulation," IEEE Trans. Veh. Technol., vol. 57, no. 4, pp. 2228-2241, Jul. 2008.

[35] S. Hong, M. Sagong, C. Lim, K. Cheun, and S. Cho, "FQAM : A modulation scheme for beyond $4 \mathrm{G}$ cellular wireless communication systems," in Proc. IEEE GLOBECOM Workshops, Dec. 2013, pp. 25-30.

[36] Y. Ma, "Pseudo-pilot: a novel paradigm of channel estimation," IEEE Signal Process. Lett., vol. 23, no. 6, pp. 814-818, Jun. 2016.

[37] B. M. Hochwald, C. B. Peel, and A. L. Swindlehurst, "A vector-perturbation technique for near-capacity multiantenna multiuser communication-part II: perturbation," IEEE Trans. Commun., vol. 53 , no. 3, pp. 537-544, Mar. 2005.

[38] L. Zheng and D. N. C. Tse, "Diversity and multiplexing: a fundamental tradeoff in multiple-antenna channels," IEEE Trans. Inf. Theory, vol. 49, no. 5, pp. 1073-1096, May 2003. 
[39] M. K. Simon and M.-S. Alouini, Digital communication over fading channels. John Wiley \& Sons, 2000.

[40] R. Xu and F. Lau, "Performance analysis for MIMO systems using zero forcing detector over fading channels," IEE Proc.-Commun., vol. 153, pp. 74-80, Feb. 2006

[41] K. Cho and D. Yoon, "On the general BER expression of one- and twodimensional amplitude modulations," IEEE Trans. Commun., vol. 50, no. 7, pp. 1074-1080, Jul. 2002.

[42] D. Tse and S. Hanly, "Linear multuser receivers: effective interference, effective bandwidth and user capacity," IEEE Trans. Inf. Theory, vol. 45, no. 3, pp. 641-657, Mar. 1999.

[43] "LTE evolved universal terrestrial radio access (E-UTRA) 'user equipment (UE) radio tranmission and reception'," 3GPP TS 36.101 v10.0.0, Tech. Rep., 2010.

[44] P. Koopman and T. Chakravarty, "Cyclic redundancy code (CRC) polynomial selection for embedded networks," in Proc IEEE Int. Conf. Dependable Syst. and Networks, Jun. 2004, pp. 145-154.

[45] "Multiplexing and channel coding," 3GPP TS 36.212 V14.4.0, 3GPP Technical Specification Group Radio Access Network; Evolved Universal Terrestrial Radio Access (E-UTRA), Tech. Rep., Sep. 2017.

[46] F. Tosato and P. Bisaglia, "Simplified soft-output demapper for binary interleaved COFDM with application to HIPERLAN/2" in Proc. IEEE Int. Conf. Commun. (ICC 2002), vol. 2, 2002, pp. 664-668.

[47] B. Hassibi and B. M. Hochwald, "How much training is needed in multiple-antenna wireless links?" IEEE Trans. Inf. Theory, vol. 49, no. 4 pp. 951-963, Apr. 2003.

[48] A. Lozano, R. Heath, and J. Andrews, "Fundamental limits of cooperation," IEEE Trans. Inf. Theory, vol. 59, no. 9, pp. 5213-5226, Sep. 2013.

[49] D. A. Basnayaka, P. J. Smith, and P. A. Martin, "Performance analysis of macrodiversity MIMO systems with MMSE and ZF receivers in flat Rayleigh fading," IEEE Trans. Wireless Commun., vol. 12, no. 5, pp. 2240-2251, May 2013.

[50] S. L. Loyka, "Channel capacity of MIMO architecture using the exponential correlation matrix," IEEE Commun. Lett., vol. 5, no. 9, pp. 369-371, Sep. 2001

[51] S. Sun, T. S. Rappaport, S. Rangan, T. A. Thomas, A. Ghosh, I. Z Kovacs, I. Rodriguez, O. Koymen, A. Partyka, and J. Jarvelainen, "Propagation path loss models for $5 \mathrm{G}$ urban micro- and macro-cellular scenarios," in Proc. IEEE 83rd Veh. Technol. Conf. (VTC Spring), May 2016, pp. 1-6. 